(n)

trobertivier Journal of Nonlinear Mathematical Physics

\title{
A hierarchy of long wave-short wave type equations: quasi-periodic behavior of solutions and their representation
}

Xianguo Geng, Yunyun Zhai, Bo Xue, Jiao Wei

To cite this article: Xianguo Geng, Yunyun Zhai, Bo Xue, Jiao Wei (2019) A hierarchy of long wave-short wave type equations: quasi-periodic behavior of solutions and their representation, Journal of Nonlinear Mathematical Physics 26:1, 1-23, DOI:

https://doi.org/10.1080/14029251.2019.1544785

To link to this article: https://doi.org/10.1080/14029251.2019.1544785

Published online: 04 January 2021 


\title{
A hierarchy of long wave-short wave type equations: quasi-periodic behavior of solutions and their representation
}

\author{
Xianguo Geng, Yunyun Zhai, Bo Xue and Jiao Wei \\ School of Mathematics and Statistics, Zhengzhou University, 100 Kexue Road, Zhengzhou, \\ Henan 450001, People's Republic of China \\ xggeng@zzu.edu.cn(Xianguo Geng); zhaiyy@zzu.edu.cn(Yunyun Zhai); \\ xuebo@zzu.edu.cn(BoXue);weijiaozzu@163.com(JiaoWei)
}

Received 24 January 2018

Accepted 24 May 2018

\begin{abstract}
Based on the Lenard recursion relation and the zero-curvature equation, we derive a hierarchy of long waveshort wave type equations associated with the $3 \times 3$ matrix spectral problem with three potentials. Resorting to the characteristic polynomial of the Lax matrix, a trigonal curve is defined, on which the Baker-Akhiezer function and two meromorphic functions are introduced. Analyzing some properties of the meromorphic functions, including asymptotic expansions at infinite points, we obtain the essential singularities and divisor of the Baker-Akhiezer function. Utilizing the theory of algebraic curves, quasi-periodic solutions for the entire hierarchy are finally derived in terms of the Riemann theta function.
\end{abstract}

Keywords: long wave-short wave type equations; Baker-Akhiezer function; meromorphic function; quasiperiodic solutions.

2000 Mathematics Subject Classification: 37K40, 37K20, 14H42

\section{Introduction}

Soliton equations have caught a great deal of attention for describing and explaining nonlinear phenomena in theoretical physics, fluid mechanics, nonlinear optics, plasma physics and other subjects [1]. Besides some famous equations such as the Korteweg-de Vries, sine-Gordon, Boussinesq and Kadomtsev Petviashvili equations [1,31,47,50], a lot of new models possessing great significance have been proposed, for example, the Camassa-Holm, Degasperis-Procesi, Novikov, and Geng-Xue equations etc [6,10,22, 23, 30,35-37]. As the research moves along, more and more systematic methods have been developed to solve these soliton systems [1,4,14, 17, 29, 33, 44]. For examples, the inverse scattering transformation [1, 17], the bilinear transformation methods of Hirota [29], the Bäcklund and Darboux transformations [44], algebro-geometric method [4, 14,32,33] and others $[19,42]$.

Since 1970s, various methods in a series of papers [3, 4, 7, 9, 14, 18, 24, 26, 32-34, 41, 51] were developed on the basis of the theory of hyperelliptic curves to obtain quasi-periodic solutions of soliton equations associated with $2 \times 2$ matrix spectral problems such as the $\mathrm{KdV}, \mathrm{KP}$, nonlinear Schrödinger, Camassa-Holm, Toda lattice, Ablowitz-Ladik equations and so on. However, it is the trigonal curve $[5,11,15,49]$ rather than the hyperelliptic curve that is the theoretic foundation to obtain the quasi-periodic solutions to soliton equations related to the third order spectral problems.

$\overline{{ }^{*} \text { Corresponding authors. }}$ 
Although the reduction theory of Riemann theta functions has been applied to study quasi-periodic solutions of the Boussinesq equation in a few of literature [2, 43, 45, 46, 52-55], the method is not a general scheme to construct quasi-periodic solutions of completely integrable systems. In 1999, Dickson and his partners proposed a unified framework which yields all algebro-geometric quasiperiodic solutions of the entire Boussinesq hierarchy $[12,13]$. Shortly after that, this method was generalized to deal with the modified Boussinesq and the Kaup-Kupershmidt hierarchies [20,21] based on the trigonal curve introduced by the characteristic polynomial of the Lax matrix. The trigonal curves in $[12,13,20,21]$ have one collective feature, that is, one infinite point which is a branch point with the triple root. Recently, the research has developed to get the quasi-periodic solutions to the coupled modified Korteweg-de Vries hierarchy, the three wave resonant interaction hierarchy and the four-component AKNS soliton hierarchy associated with the trigonal curves having three different infinite points which are not branch points [25, 28, 39, 40].

In this paper, we first derive a hierarchy of long wave-short wave type equations associated with the $3 \times 3$ matrix spectral problem with three potentials. The first nontrivial member in the hierarchy is the long wave-short wave type system

$$
\begin{aligned}
u_{t} & =2(v w)_{x}, \\
v_{t} & =v_{x x}-u v_{x}, \\
w_{t} & =-w_{x x}-u w_{x} .
\end{aligned}
$$

Equation (1.1) reduces to

$$
u_{t}=2\left(|v|^{2}\right)_{x}, \quad v_{t}=-i v_{x x}-u v_{x}
$$

if $x \rightarrow-i x, t \rightarrow-i t, w=v^{*}$, which is different from the standard long wave-short wave resonance system $[8,38,56]$. Another principal subject of the present paper is to construct quasi-periodic solutions for the long wave-short wave type hierarchy on the basis of the theory of algebraic curves. To this end, one introduces the trigonal curve with the aid of the characteristic polynomial of the Lax matrix. A distinguishing feature for the trigonal curve associated with the hierarchy is that it has two infinite points, one of which is a double branch point and the other is not a branch point. Compared with references [12, 13, 20, 21, 25, 28, 39, 40], the trigonal curve in this paper is more general. Therefore, we need to reinvestigate the local coordinates near infinite points, the Abelian differentials and other basic properties.

The outline of this paper is as follows. In section 2 , we consider a $3 \times 3$ matrix spectral problem with three potentials and derive a hierarchy of nonlinear evolution equations with the aid of three sets of Lenard recursion equations and the stationary zero-curvature equation. In section 3 , a trigonal curve is introduced by using the characteristic polynomial of the Lax matrix, on which the Baker-Akhiezer function together with two related meromorphic functions is given. Based on the asymptotic properties of the two meromorphic functions near the infinite points, the essential singularities of Baker-Akhiezer function are derived. Accordingly, the Abelian differentials of the second kind are given for the purpose of representation. In section 4, we investigate some properties of the two meromorphic functions and obtain the Dubrovin-type equations. Subsequently, we derive divisors of meromorphic functions and the Baker-Akhiezer function which are necessary for the construction of the quasi-periodic solutions. Section 5 finally constructs the Riemann theta function representations for the long wave-short wave type hierarchy according to the asymptotic properties and the quasi-periodic characters of the meromorphic function and the Baker-Akhiezer function. 


\section{A long wave-short wave type hierarchy}

In this section, we shall derive a hierarchy of long wave-short wave type models. To this end, we consider a $3 \times 3$ matrix spectral problem with three potentials

$$
\psi_{x}=U \psi, \quad \psi=\left(\begin{array}{l}
\psi_{1} \\
\psi_{2} \\
\psi_{3}
\end{array}\right), \quad U=\left(\begin{array}{ccc}
u & \lambda & v \\
1 & 0 & 0 \\
w & 0 & 0
\end{array}\right)
$$

where $u, v, w$ are three potentials, and $\lambda$ a constant spectral parameter. We first introduce three sets of Lenard recursion equations:

$$
\begin{aligned}
& K s_{j}=J s_{j+1}, \quad j \geq 0,\left.\quad s_{j}\right|_{(u, v, w)=0}=0, \quad j \geq 1, \\
& K \hat{s}_{j}=J \hat{s}_{j+1}, \quad j \geq 0,\left.\quad \hat{s}_{j}\right|_{(u, v, w)=0}=0, \quad j \geq 1, \\
& K \tilde{s}_{j}=J \tilde{s}_{j+1}, \quad j \geq 0,\left.\quad \tilde{s}_{j}\right|_{(u, v, w)=0}=0, \quad j \geq 1,
\end{aligned}
$$

where the two operators are defined as

$$
\begin{gathered}
K=\left(\begin{array}{ccccc}
-v w \partial & \partial u-\partial^{2} & w \partial & \partial v+v \partial & 0 \\
u v \partial-\partial v \partial & \partial v-v \partial & \partial^{2}-u \partial & v^{2} & -v \\
0 & \partial w+w \partial & 0 & -\partial^{2}-u \partial-v w & w \\
-\partial^{2}-u \partial & 2 \partial & w & -v & 0 \\
v w \partial & 0 & -w \partial & -v \partial & \partial
\end{array}\right) \\
J=\left(\begin{array}{ccccc}
-2 \partial & 0 & 0 & 0 & 0 \\
-v & 0 & 1 & 0 & 0 \\
w & 0 & 0 & -1 & 0 \\
-\partial^{2}-u \partial & 2 \partial & w & -v & 0 \\
v w \partial & 0 & -w \partial & -v \partial & \partial
\end{array}\right)
\end{gathered}
$$

and the starting points $s_{0}=(0,0,0,0,1)^{T}, \hat{s}_{0}=(0,1,0,0,0)^{T}, \tilde{s}_{0}=(1,0, v, w, v w)^{T}$. Then the sequences $s_{j}, \hat{s}_{j}, \tilde{s}_{j}$ can be determined uniquely. For example, the first three members read as

$$
s_{1}=\left(\begin{array}{c}
0 \\
0 \\
-v \\
-w \\
-v w
\end{array}\right), \quad \hat{s}_{1}=\left(\begin{array}{c}
-\frac{1}{2} u \\
-\frac{1}{4} u_{x}-\frac{1}{8} u^{2}-\frac{1}{2} v w \\
v_{x}-\frac{1}{2} u v \\
-w_{x}-\frac{1}{2} u w \\
v_{x} w-v w_{x}-\frac{1}{2} u v w
\end{array}\right), \quad \tilde{s}_{1}=\left(\begin{array}{c}
-v w \\
-v_{x} w \\
v_{x x}-u v_{x}-v^{2} w \\
w_{x x}+u w_{x}-v w^{2} \\
\tilde{s}_{1}^{(5)}
\end{array}\right),
$$

where

$$
\tilde{s}_{1}^{(5)}=v_{x x} w-v_{x} w_{x}+v w_{x x}-u v_{x} w+u v w_{x}-v^{2} w^{2} .
$$

In order to generate a hierarchy of nonlinear evolution equations associated with the spectral problem (2.1), we define a $3 \times 3$ matrix

$$
V=\left(V_{i j}\right)_{3 \times 3}=\left(\begin{array}{lll}
V_{11} & \lambda V_{12} & V_{13} \\
V_{21} & \lambda V_{22} & V_{23} \\
V_{31} & \lambda V_{32} & V_{33}
\end{array}\right)
$$


with the elements

$$
\begin{aligned}
& V_{11}=(u-\partial) c+v e+\lambda b, \quad V_{12}=c, \quad V_{13}=-v b_{x}+v c+d_{x}, \quad V_{21}=c-b_{x}, \\
& V_{22}=b, \quad V_{23}=d, \quad V_{31}=w c-e_{x}, \quad V_{32}=e, \quad V_{33}=f .
\end{aligned}
$$

Then the stationary zero-curvature equation

$$
V_{x}-[U, V]=0
$$

is equivalent to

$$
\begin{aligned}
& -v w \partial b+\left(\partial u-\partial^{2}\right) c+w \partial d+(\partial v+v \partial) e+2 \lambda \partial b=0, \\
& (u v \partial-\partial v \partial) b+(\partial v-v \partial) c+\left(\partial^{2}-u \partial\right) d+v^{2} e-v f+\lambda(v b-d)=0, \\
& (\partial w+w \partial) c-\left(\partial^{2}+u \partial+v w\right) e+w f+\lambda(-w b+e)=0, \\
& -\left(\partial^{2}+u \partial\right) b+2 \partial c+w d-v e=0, \\
& v w \partial b-w \partial d-v \partial e+\partial f=0 .
\end{aligned}
$$

Expanding $b, c, d, e, f$ into the Laurent polynomials in $\lambda$ :

$$
(b, c, d, e, f)=\sum_{j \geq 0}\left(b_{j}, c_{j}, d_{j}, e_{j}, f_{j}\right) \lambda^{-j},
$$

equation (2.8) is equivalent to the following recursion equations

$$
K S_{j}=J S_{j+1}, \quad j \geq 0, \quad J S_{0}=0,
$$

where $S_{j}=\left(b_{j}, c_{j}, d_{j}, e_{j}, f_{j}\right)^{T}$. Since equation $J S_{0}=0$ has a general solution

$$
S_{0}=\alpha_{0} s_{0}+\beta_{0} \hat{s}_{0}+\delta_{0} \tilde{s_{0}},
$$

then functions $S_{j}$ given by

$$
S_{j}=\sum_{l=0}^{j}\left(\alpha_{l} s_{j-l}+\beta_{l} \hat{s}_{j-l}+\delta_{l} \tilde{s}_{j-l}\right),
$$

satisfy the recursion equation (2.10), where $\alpha_{j}, \beta_{j}, \delta_{j}$ are arbitrary constants.

Let $\psi$ satisfy the spectral problem (2.1) and the auxiliary problem

$$
\psi_{t_{r}}=\widetilde{V}^{(r)} \psi, \quad \widetilde{V}^{(r)}=\left(\widetilde{V}_{i j}^{(r)}\right)_{3 \times 3},
$$

where $\widetilde{V}_{i j}^{(r)}=V_{i j}\left(\tilde{b}^{(r)}, \tilde{c}^{(r)}, \tilde{d}^{(r)}, \tilde{e}^{(r)}, \tilde{f}^{(r)}\right)$,

$$
\left(\tilde{b}^{(r)}, \tilde{c}^{(r)}, \tilde{d}^{(r)}, \tilde{e}^{(r)}, \tilde{f}^{(r)}\right)=\sum_{j \geq 0}^{r}\left(\tilde{b}_{j}, \tilde{c}_{j}, \tilde{d}_{j}, \tilde{e}_{j}, \tilde{f}_{j}\right) \lambda^{r-j},
$$

with $\tilde{S}_{j}=\left(\tilde{b}_{j}, \tilde{c}_{j}, \tilde{d}_{j}, \tilde{e}_{j}, \tilde{f}_{j}\right)^{T}$ determined by

$$
\tilde{S}_{j}=\sum_{l=0}^{j}\left(\tilde{\alpha}_{l} s_{j-l}+\tilde{\beta}_{l} \hat{s}_{j-l}+\tilde{\delta}_{l} \tilde{s}_{j-l}\right) .
$$

The constants $\tilde{\alpha}_{j}, \tilde{\beta}_{j}, \tilde{\delta}_{j}$ here are independent of the choice of $\alpha_{j}, \beta_{j}, \delta_{j}$. Then the compatibility condition of (2.1) and (2.13) yields the zero-curvature equation, $U_{t_{r}}-\widetilde{V}_{x}^{(r)}+\left[U, \widetilde{V}^{(r)}\right]=0$, which is 
equivalent to a hierarchy of nonlinear evolution equations

$$
\left(u_{t_{r}}, v_{t_{r}}, w_{t_{r}}\right)^{T}=X_{r}, \quad r \geq 0,
$$

where the vector fields $X_{r}=P\left(K \tilde{S}_{r}\right)=P\left(J \tilde{S}_{r+1}\right), P$ is the projective map $P\left(\gamma^{1}, \gamma^{2}, \gamma^{3}, \gamma^{4}, \gamma^{5}\right)^{T}$ $\rightarrow\left(\gamma^{1}, \gamma^{2}, \gamma^{3}\right)^{T}$. The first member in the hierarchy for $r=0$ is a long wave-short wave type system

$$
\begin{aligned}
u_{t_{0}} & =\tilde{\beta}_{0} u_{x}+2 \tilde{\delta}_{0}(v w)_{x}, \\
v_{t_{0}} & =-\tilde{\alpha}_{0} v+\tilde{\beta}_{0} v_{x}+\tilde{\delta}_{0}\left(v_{x x}-u v_{x}\right), \\
w_{t_{0}} & =\tilde{\alpha}_{0} w+\tilde{\beta}_{0} w_{x}+\tilde{\delta}_{0}\left(-w_{x x}-u w_{x}\right) .
\end{aligned}
$$

As $\tilde{\alpha}_{0}=\tilde{\beta}_{0}=0, \tilde{\delta}_{0}=1, t_{0}=t$, equation (2.17) turns into (1.1). For $r=1$, the second member in the hierarchy (2.16) reads as

$$
\begin{aligned}
& u_{t_{1}}=- 2 \tilde{\alpha}_{0}(v w)_{x}+\tilde{\beta}_{0}\left(\frac{1}{4} u_{x x x}+\frac{3}{2} v_{x x} w-\frac{3}{2} v w_{x x}-\frac{3}{2}(u v w)_{x}-\frac{3}{8} u^{2} u_{x}\right) \\
&+2 \tilde{\delta}_{0}\left(v_{x x} w+v w_{x x}+u v w_{x}-u v_{x} w-v^{2} w^{2}\right)_{x}+\tilde{\beta}_{1} u_{x}+2 \tilde{\delta}_{1}(v w)_{x}, \\
& v_{t_{1}}=\tilde{\alpha}_{0}\left(-v_{x x}+u v_{x}\right)+\tilde{\beta}_{0}\left(v_{x x x}-\frac{3}{4} u_{x} v_{x}-\frac{3}{2} u v_{x x}-\frac{3}{2} v v_{x} w+\frac{3}{8} u^{2} v_{x}\right)+\tilde{\delta}_{0}\left(v_{x x x x}-u_{x x} v_{x}-2 u_{x} v_{x x}-2 u v_{x x x}-2 v_{x}^{2} w-2 v v_{x x} w+u u_{x} v_{x}\right. \\
&\left.+u^{2} v_{x x}+2 u v v_{x} w\right)-\tilde{\alpha}_{1} v+\tilde{\beta}_{1} v_{x}+\tilde{\delta}_{1}\left(v_{x x}-u v_{x}\right) \\
& w_{t_{1}}=\tilde{\alpha}_{0}\left(w_{x x}+u w_{x}\right)+\tilde{\beta}_{0}\left(w_{x x x}-\frac{3}{2} v w w_{x}+\frac{3}{2} u w_{x x}+\frac{3}{4} u_{x} w_{x}+\frac{3}{8} u^{2} w_{x}\right) \\
&+\tilde{\delta}_{0}\left(-w_{x x x x}-u_{x x} w_{x}-2 u_{x} w_{x x}-2 u w_{x x x}-u u_{x} w_{x}-u^{2} w_{x x}+2 v w_{x}^{2}\right. \\
&\left.+2 v w w_{x x}+2 u v w w_{x}\right)+\tilde{\alpha}_{1} w+\tilde{\beta}_{1} w_{x}+\tilde{\delta}_{1}\left(-w_{x x}-u w_{x}\right) .
\end{aligned}
$$

If choosing $\tilde{\beta}_{0}=1, \tilde{\alpha}_{0}=\tilde{\alpha}_{1}=\tilde{\beta}_{1}=\tilde{\delta}_{0}=\tilde{\delta}_{1}=0$ or $\tilde{\delta}_{0}=1, \tilde{\alpha}_{0}=\tilde{\beta}_{0}=\tilde{\alpha}_{1}=\tilde{\beta}_{1}=\tilde{\delta}_{1}=0$, then (2.18) is respectively reduced to a new coupled $\mathrm{mKdV}$ equation

$$
\begin{aligned}
u_{t_{1}} & =\frac{1}{4} u_{x x x}-\frac{3}{8} u^{2} u_{x}+\frac{3}{2} v_{x x} w-\frac{3}{2} v w_{x x}-\frac{3}{2}(u v w)_{x}, \\
v_{t_{1}} & =v_{x x x}-\frac{3}{4} u_{x} v_{x}-\frac{3}{2} u v_{x x}-\frac{3}{2} v v_{x} w+\frac{3}{8} u^{2} v_{x}, \\
w_{t_{1}} & =w_{x x x}+\frac{3}{4} u_{x} w_{x}+\frac{3}{2} u w_{x x}-\frac{3}{2} v w w_{x}+\frac{3}{8} u^{2} w_{x},
\end{aligned}
$$

or

$$
\begin{gathered}
u_{t_{1}}=2\left(v_{x x} w+v w_{x x}+u v w_{x}-u v_{x} w-v^{2} w^{2}\right)_{x} \\
v_{t_{1}}=v_{x x x x}-u_{x x} v_{x}-2 u_{x} v_{x x}-2 u v_{x x x}-2 v_{x}^{2} w-2 v v_{x x} w \\
\quad+u u_{x} v_{x}+u^{2} v_{x x}+2 u v v_{x} w \\
\begin{array}{c}
w_{t_{1}}=-w_{x x x x}-u_{x x} w_{x}-2 u_{x} w_{x x}-2 u w_{x x x}-u u_{x} w_{x}-u^{2} w_{x x} \\
+2 v w_{x}^{2}+2 v w w_{x x}+2 u v w w_{x} .
\end{array}
\end{gathered}
$$

\section{Meromorphic functions and Baker-Akhiezer function}

In this section, we shall define a trigonal curve $\mathscr{K}_{m-1}$, the vector Baker-Akhiezer function and two meromorphic functions on $\mathscr{K}_{m-1}$. The Abelian differentials of the second kind are introduced on the basis of the analysis for Baker-Akhiezer function $\psi_{2}$ at infinite points. 
With the help of the $n$-th stationary flow, we introduce a Lax matrix $V^{(n)}=\left(V_{i j}^{(n)}\right)_{3 \times 3}=$ $\left(\left(\lambda^{n} V_{i j}\right)_{+}\right)_{3 \times 3}$, which satisfies the Lax equation

$$
\begin{aligned}
V_{x}^{(n)}-\left[U, V^{(n)}\right] & =0, \\
V_{t_{r}}^{(n)}-\left[\widetilde{V}^{(r)}, V^{(n)}\right] & =0 .
\end{aligned}
$$

Then the characteristic polynomial $\mathscr{F}_{m}(\lambda, y)=\operatorname{det}\left(y I-V^{(n)}\right)$ of the Lax matrix $V^{(n)}$ is a polynomial of $\lambda$ independent of variables $x$ and $t_{r}$, which can be expressed in the following form

$$
\operatorname{det}\left(y I-V^{(n)}\right)=y^{3}-y^{2} R_{m}(\lambda)+y S_{m}(\lambda)-T_{m}(\lambda),
$$

where $R_{m}(\lambda), S_{m}(\lambda)$ and $T_{m}(\lambda)$ are polynomials with constant coefficients of $\lambda$

$$
\begin{aligned}
R_{m}(\lambda) & =V_{11}^{(n)}+\lambda V_{22}^{(n)}+V_{33}^{(n)}=2 \delta_{0} \lambda^{n+1}+\left(2 \delta_{1}+\alpha_{0}\right) \lambda^{n}+\cdots \\
S_{m}(\lambda) & =\left|\begin{array}{ll}
V_{11}^{(n)} & \lambda V_{12}^{(n)} \\
V_{21}^{(n)} & \lambda V_{22}^{(n)}
\end{array}\right|+\left|\begin{array}{cc}
V_{11}^{(n)} & V_{13}^{(n)} \\
V_{31}^{(n)} & V_{33}^{(n)}
\end{array}\right|+\left|\begin{array}{cc}
\lambda V_{22}^{(n)} & V_{23}^{(n)} \\
\lambda V_{32}^{(n)} & V_{33}^{(n)}
\end{array}\right| \\
& =\delta_{0}^{2} \lambda^{2 n+2}+\left(2 \delta_{0} \delta_{1}+2 \alpha_{0} \delta_{0}-\beta_{0}^{2}\right) \lambda^{2 n+1}+\cdots \\
T_{m}(\lambda) & =\left|\begin{array}{lll}
V_{11}^{(n)} & \lambda V_{12}^{(n)} & V_{13}^{(n)} \\
V_{21}^{(n)} & \lambda V_{22}^{(n)} & V_{23}^{(n)} \\
V_{31}^{(n)} & \lambda V_{32}^{(n)} & V_{33}^{(n)}
\end{array}\right|=\lambda\left[\alpha_{0} \delta_{0}^{2} \lambda^{3 n+1}+\left(2 \alpha_{0} \delta_{0} \delta_{1}+\alpha_{1} \delta_{0}^{2}-\alpha_{0} \beta_{0}^{2}\right) \lambda^{3 n}+\cdots\right]
\end{aligned}
$$

This naturally leads to a trigonal curve $\mathscr{K}_{m-1}$ of degree $m=3 n+2$ with respect to $\lambda$ by

$$
\mathscr{K}_{m-1}: \quad \mathscr{F}_{m}(\lambda, y)=y^{3}-y^{2} R_{m}(\lambda)+y S_{m}(\lambda)-T_{m}(\lambda)=0 .
$$

According to (3.4) and (3.5), the trigonal curve $\mathscr{K}_{m-1}$ can be compactified by adding two infinite points $P_{\infty_{1}}$ and $P_{\infty_{2}}$ for which we take $P_{\infty_{1}}$ as a double point without loss of generality. $\mathscr{K}_{m-1}$ is nonsingular or smooth means that for every point $Q_{0}=\left(\lambda_{0}, y_{0}\right) \in \mathscr{K}_{m-1} \backslash\left\{P_{\infty_{1}}, P_{\infty_{2}}\right\}$, $\left.\left(\frac{\partial \mathscr{F}_{m}}{\partial \lambda}, \frac{\partial \mathscr{F}_{m}}{\partial y}\right)\right|_{(\lambda, y)=\left(\lambda_{0}, y_{0}\right)} \neq 0$. Adding the assumption of irreducibility, the trigonal curve $\mathscr{K}_{m-1}$ becomes connected. For the sake of convenience, we use the same symbol $\mathscr{K}_{m-1}$ in the following text to denote the three sheeted nonsingular compact Riemann surface. Obviously, the discriminant of (3.5) is $\Delta(\lambda)=-R_{m}^{2} S_{m}^{2}+4 R_{m}^{3} T_{m}+4 S_{m}^{3}-18 R_{m} S_{m} T_{m}+27 T_{m}^{2}=-4 \beta_{0}^{2} \delta_{0}^{4} \lambda^{6 n+5}+\cdots$, which has at most $6 n+5$ zeros. Therefore, the Riemann-Hurwitz formula shows that the arithmetic genus of $\mathscr{K}_{m-1}$ is $3 n+1$ for $\beta_{0} \delta_{0} \neq 0[16,27,48]$.

Equip the Riemann surface $\mathscr{K}_{m-1}$ with homology basis $\left\{\mathfrak{a}_{j}, \mathfrak{b}_{j}\right\}_{j=1}^{m-1}$, which are independent and have intersection numbers as follows

$$
\mathrm{a}_{j} \circ \mathfrak{b}_{k}=\delta_{j k}, \quad \mathrm{a}_{j} \circ \mathrm{a}_{k}=0, \quad \mathfrak{b}_{j} \circ \mathfrak{b}_{k}=0, \quad j, k=1, \ldots, m-1 .
$$

For the present, we will choose as our basis the following set

$$
\varpi_{l}(P)=\frac{1}{3 y^{2}(P)-2 R_{m}(\lambda) y(P)+S_{m}(\lambda)} \begin{cases}\lambda^{l-1} d \lambda, & 1 \leq l \leq 2 n+1, \\ y(P) \lambda^{l-2 n-2} d \lambda, & 2 n+2 \leq l \leq 3 n+1,\end{cases}
$$


which are $3 n+1$ linearly independent holomorphic differentials on $\mathscr{K}_{m-1}$. By using the homology basis $\left\{\mathrm{a}_{j}\right\}_{j=1}^{m-1}$ and $\left\{\mathrm{b}_{j}\right\}_{j=1}^{m-1}$, the period matrices $A=\left(A_{j k}\right)$ and $B=\left(B_{j k}\right)$ can be constructed from

$$
A_{j k}=\int_{\mathfrak{a}_{k}} \varpi_{j}, \quad B_{j k}=\int_{\mathbb{b}_{k}} \varpi_{j}
$$

It is possible to show that the matrices $A$ and $B$ are invertible $[16,27,48]$. Now we define the matrices $C$ and $\tau$ by $C=A^{-1}, \tau=A^{-1} B$. The matrix $\tau$ can be shown to be symmetric $\left(\tau_{j k}=\tau_{k j}\right)$ and has a positive-definite imaginary part $(\operatorname{Im} \tau>0)$. If we normalized $\varpi_{l}(P)$ into new basis $\underline{\omega}=$ $\left(\omega_{1}, \ldots, \omega_{m-1}\right)$

$$
\omega_{j}=\sum_{l=1}^{m-1} C_{j l} \bar{\varpi}_{l}
$$

then we have $\int_{\mathrm{a}_{k}} \omega_{j}=\delta_{j k}, \int_{\mathbb{b}_{k}} \omega_{j}=\tau_{j k}, j, k=1, \ldots, m-1$.

The complex structure on $\mathscr{K}_{m-1}$ is defined in the usual way by introducing local coordinates $\zeta_{Q_{0}}: P \rightarrow\left(\lambda-\lambda_{0}\right)$ near points $Q_{0}=\left(\lambda_{0}, y\left(Q_{0}\right)\right) \in \mathscr{K}_{m-1}$ which are not branch points nor infinite points of $\mathscr{K}_{m-1}, \zeta_{P_{\infty_{j}}}: P \rightarrow \lambda^{-1 /(3-j)}$ near the points $P_{\infty_{j}} \in \mathscr{K}_{m-1}, j=1,2$, and similar at others branch points of $\mathscr{K}_{m-1}$.

Given these preliminaries, let $\psi\left(P, x, x_{0}, t_{r}, t_{0, r}\right)$ denote the vector Baker-Akhiezer function by

$$
\begin{aligned}
& \psi_{x}\left(P, x, x_{0}, t_{r}, t_{0, r}\right)=U\left(u\left(x, t_{r}\right), v\left(x, t_{r}\right), w\left(x, t_{r}\right) ; \lambda(P)\right) \psi\left(P, x, x_{0}, t_{r}, t_{0, r}\right), \\
& \psi_{t_{r}}\left(P, x, x_{0}, t_{r}, t_{0, r}\right)=\widetilde{V}^{(r)}\left(u\left(x, t_{r}\right), v\left(x, t_{r}\right), w\left(x, t_{r}\right) ; \lambda(P)\right) \psi\left(P, x, x_{0}, t_{r}, t_{0, r}\right), \\
& V^{(n)}\left(u\left(x, t_{r}\right), v\left(x, t_{r}\right), w\left(x, t_{r}\right) ; \lambda(P)\right) \psi\left(P, x, x_{0}, t_{r}, t_{0, r}\right)=y(P) \psi\left(P, x, x_{0}, t_{r}, t_{0, r}\right), \\
& \psi_{2}\left(P, x_{0}, x_{0}, t_{0, r}, t_{0, r}\right)=1, \quad x, t_{r} \in \mathbb{C}, \quad P \in \mathscr{K}_{m-1} \backslash\left\{P_{\infty_{1}}, P_{\infty_{2}}\right\} .
\end{aligned}
$$

Define two meromorphic functions $\phi_{2}\left(P, x, t_{r}\right)$ and $\phi_{3}\left(P, x, t_{r}\right)$ on $\mathscr{K}_{m-1}$ closely related to the BakerAkhiezer function by

$$
\begin{array}{ll}
\phi_{2}\left(P, x, t_{r}\right)=\frac{\psi_{1}\left(P, x, x_{0}, t_{r}, t_{0, r}\right)}{\psi_{2}\left(P, x, x_{0}, t_{r}, t_{0, r}\right)}, & P \in \mathscr{K}_{m-1}, x, t_{r} \in \mathbb{C}, \\
\phi_{3}\left(P, x, t_{r}\right)=\frac{\psi_{1}\left(P, x, x_{0}, t_{r}, t_{0, r}\right)}{\psi_{3}\left(P, x, x_{0}, t_{r}, t_{0, r}\right)}, & P \in \mathscr{K}_{m-1}, x, t_{r} \in \mathbb{C} .
\end{array}
$$

Lemma 3.1. Assume that (3.9), (3.10), (3.11) hold and let $P=(\lambda, y(P)) \in \mathscr{K}_{m-1} \backslash\left\{P_{\infty_{1}}, P_{\infty_{2}}\right\}$ and $\left(\lambda, x, t_{r}\right) \in \mathbb{C}^{3}$. Then meromorphic functions $\phi_{2}\left(P, x, t_{r}\right)$ and $\phi_{3}\left(P, x, t_{r}\right)$ have the following asymptotic expansions near $P_{\infty_{j}} \in \mathscr{K}_{m-1}, j=1,2$, under the local coordinate $\zeta=\lambda^{-1 /(3-j)}$

$$
\begin{aligned}
& \phi_{2}\left(P, x, t_{r}\right) \underset{\zeta \rightarrow 0}{=}\left\{\begin{array}{c}
\zeta^{-1}+\frac{u}{2}+\frac{1}{8}\left(-2 u_{x}+u^{2}+4 v w\right) \zeta+\frac{1}{8}\left(-u u_{x}-2 v_{x} w-6 v w_{x}+u_{x x}\right) \zeta^{2}+O\left(\zeta^{3}\right), \\
\text { as } P \rightarrow P_{\infty_{1}}, \quad \zeta=\lambda^{-1 / 2}, \\
\frac{v_{x}}{v}+\frac{1}{v^{2}}\left[v v_{x x x}-v_{x} v_{x x}-\left(u v_{x}\right)_{x} v+u v_{x}^{2}-v_{x} v^{2} w\right] \zeta+O\left(\zeta^{2}\right), \\
\text { as } P \rightarrow P_{\infty_{2}}, \quad \zeta=\lambda^{-1},
\end{array}\right. \\
& \phi_{3}\left(P, x, t_{r}\right) \underset{\zeta \rightarrow 0}{=}\left\{\begin{array}{lll}
\frac{1}{w} \zeta^{-1}+O(1), & \text { as } P \rightarrow P_{\infty_{1}}, & \zeta=\lambda^{-1 / 2} \\
-v_{x} \zeta+O\left(\zeta^{2}\right), & \text { as } P \rightarrow P_{\infty_{2}}, & \zeta=\lambda^{-1}
\end{array}\right.
\end{aligned}
$$


Proof. Expressions (3.9) and (3.10) imply that meromorphic functions $\phi_{2}\left(P, x, t_{r}\right)$ satisfies the Riccati-type equation

$$
\phi_{2, x x}+3 \phi_{2} \phi_{2, x}+\phi_{2}^{3}-\left(\frac{v_{x}}{v}+u\right)\left(\phi_{2, x}+\phi_{2}^{2}\right)=\left(u_{x}-\frac{u v_{x}}{v}+v w\right) \phi_{2}+\lambda\left(\phi_{2}-\frac{v_{x}}{v}\right) .
$$

We can insert the two following ansatzs into the above equation (3.14)

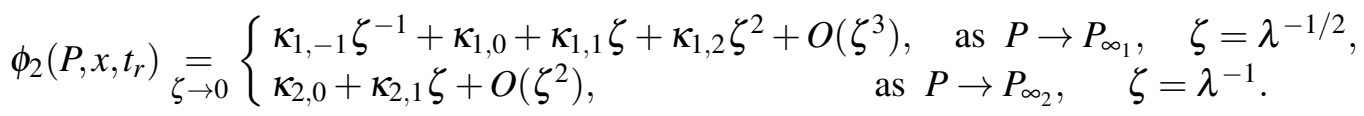

A comparison of the same powers of $\zeta$ then proves the first expression (3.12) in this lemma. The first expression in (3.9) implies the relationship between $\phi_{2}$ and $\phi_{3}$

$$
\phi_{3}=\frac{v \phi_{2}}{\phi_{2, x}+\phi_{2}^{2}-u \phi_{2}-\lambda} .
$$

Utilizing the expansions of $\phi_{2}$ in (3.12), we can easily derive (3.13).

Taking advantage of (3.9), (3.12) and (3.13), we can calculate out the asymptotic behaviors of $y(P)$ near $P_{\infty_{1}}, P_{\infty_{2}}$ as

$$
y(P) \underset{\zeta \rightarrow 0}{=}\left\{\begin{array}{l}
\zeta^{-2 n-2}\left(\delta_{0}+\beta_{0} \zeta+\delta_{1} \zeta^{2}+\beta_{1} \zeta^{3}+O\left(\zeta^{4}\right)\right), \text { as } P \rightarrow P_{\infty_{1}}, \quad \zeta=\lambda^{-1 / 2} \\
\zeta^{-n}\left(\alpha_{0}+\alpha_{1} \zeta+O\left(\zeta^{2}\right)\right), \text { as } P \rightarrow P_{\infty_{2}}, \quad \zeta=\lambda^{-1}
\end{array}\right.
$$

Subsequently, one infers that

$$
\omega_{j} \underset{\zeta \rightarrow 0}{=}\left\{\begin{array}{l}
\left(\frac{-C_{j, 2 n+1}-\delta_{0} C_{j, 3 n+1}}{\beta_{0} \delta_{0}}+O(\zeta)\right) d \zeta, \quad \text { as } P \rightarrow P_{\infty_{1}}, \quad \zeta=\lambda^{-1 / 2}, \\
\left(\frac{-C_{j, 2 n+1}}{\delta_{0}^{2}}+O(\zeta)\right) d \zeta, \quad \text { as } P \rightarrow P_{\infty_{2}}, \quad \zeta=\lambda^{-1}
\end{array}\right.
$$

Furthermore, we could write $\omega_{j}$ in the following form:

$$
\omega_{k}=\sum_{l=0}^{\infty} \rho_{k, l}\left(P_{\infty_{j}}\right) \zeta^{l} d \zeta, \text { as } P \rightarrow P_{\infty_{j}}, \quad \zeta=\lambda^{-1 /(3-j)}
$$

where $\rho_{k, l}\left(P_{\infty_{j}}\right)$ are constants, $j=1,2 ; k=1,2, \ldots, 3 n+1$.

From the first two expression of (3.9), we arrive at the formula of $\psi_{2}\left(P, x, x_{0}, t_{r}, t_{0, r}\right)$ as follows

$$
\begin{gathered}
\psi_{2}\left(P, x, x_{0}, t_{r}, t_{0, r}\right)=\exp \left(\int_{x_{0}}^{x}\left[\phi_{2}\left(P, x^{\prime}, t_{r}\right)\right] d x^{\prime}+\int_{t_{0, r}}^{t_{r}}\left[\widetilde{V}_{21}^{(r)}\left(\lambda, x_{0}, t^{\prime}\right) \phi_{2}\left(P, x_{0}, t^{\prime}\right)+\lambda \widetilde{V}_{22}^{(r)}\left(\lambda, x_{0}, t^{\prime}\right)\right.\right. \\
\left.\left.+\frac{\widetilde{V}_{23}^{(r)}\left(\lambda, x_{0}, t^{\prime}\right)}{v\left(x_{0}, t^{\prime}\right)}\left(\phi_{2, x}\left(P, x_{0}, t^{\prime}\right)+\phi_{2}^{2}\left(P, x_{0}, t^{\prime}\right)-u\left(x_{0}, t^{\prime}\right) \phi_{2}\left(P, x_{0}, t^{\prime}\right)-\lambda\right)\right] d t^{\prime}\right)
\end{gathered}
$$

from which we can deduce the essential singularity of $\psi_{2}\left(P, x, x_{0}, t_{r}, t_{0, r}\right)$ near $P_{\infty_{j}}, j=1,2$. For the sake of convenience, we define a function

$$
\begin{aligned}
I_{r}\left(P, x, t_{r}\right)= & \widetilde{V}_{21}^{(r)}\left(\lambda, x, t_{r}\right) \phi_{2}\left(P, x, t_{r}\right)+\lambda \widetilde{V}_{22}^{(r)}\left(\lambda, x, t_{r}\right) \\
& +\frac{\widetilde{V}_{23}^{(r)}\left(\lambda, x, t_{r}\right)}{v\left(x, t_{r}\right)}\left(\phi_{2, x}\left(P, x, t_{r}\right)+\phi_{2}^{2}\left(P, x, t_{r}\right)-u\left(x, t_{r}\right) \phi_{2}\left(P, x, t_{r}\right)-\lambda\right)
\end{aligned}
$$


whose three homogeneous cases is denoted by

$$
\begin{aligned}
\bar{I}_{r}^{(\varepsilon)}\left(P, x, t_{r}\right) & =\overline{\widetilde{V}}_{21}^{(r, \varepsilon)}\left(\lambda, x, t_{r}\right) \phi_{2}\left(P, x, t_{r}\right)+\lambda \overline{\vec{V}}_{22}^{(r, \varepsilon)}\left(\lambda, x, t_{r}\right) \\
& +\frac{\overline{\widetilde{V}}_{23}^{(r, \varepsilon)}\left(\lambda, x, t_{r}\right)}{v\left(x, t_{r}\right)}\left(\phi_{2, x}\left(P, x, t_{r}\right)+\phi_{2}^{2}\left(P, x, t_{r}\right)-u\left(x, t_{r}\right) \phi_{2}\left(P, x, t_{r}\right)-\lambda\right), \varepsilon=1,2,3,
\end{aligned}
$$

where

$$
\begin{aligned}
& \overline{\widetilde{V}}_{i j}^{(r, 1)}=\left.\widetilde{V}_{i j}^{(r)}\right|_{\tilde{\alpha}_{0}=1, \tilde{\alpha}_{1}=\cdots=\tilde{\alpha}_{r}=\tilde{\beta}_{0}=\cdots=\tilde{\beta}_{r}=\tilde{\delta}_{0}=\cdots=\tilde{\delta}_{r}=0}, \\
& \overline{\widetilde{V}}_{i j}^{(r, 2)}=\left.\widetilde{V}_{i j}^{(r)}\right|_{\tilde{\beta}_{0}=1, \tilde{\alpha}_{0}=\cdots=\tilde{\alpha}_{r}=\tilde{\beta}_{1}=\cdots=\tilde{\beta}_{r}=\tilde{\delta}_{0}=\cdots=\tilde{\delta}_{r}=0}, \\
& \overline{\widetilde{V}}_{i j}^{(r, 3)}=\left.\widetilde{V}_{i j}^{(r)}\right|_{\tilde{\delta}_{0}=1, \tilde{\alpha}_{0}=\cdots=\tilde{\alpha}_{r}=\tilde{\beta}_{0}=\cdots=\tilde{\beta}_{r}=\tilde{\delta}_{1}=\cdots=\tilde{\delta}_{r}=0} .
\end{aligned}
$$

Homogeneous polynomials $\overline{\tilde{b}}^{(r, \varepsilon)}, \overline{\tilde{c}}^{(r, \varepsilon)}, \overline{\tilde{d}}^{(r, \varepsilon)}, \overline{\tilde{e}}^{(r, \varepsilon)}, \overline{\tilde{f}}^{(r, \varepsilon)}$ and $\overline{\tilde{b}}_{j}^{(\varepsilon)}, \overline{\tilde{c}}_{j}^{(\varepsilon)}, \overline{\tilde{d}}_{j}^{(\varepsilon)}, \overline{\tilde{e}}_{j}^{(\varepsilon)}, \overline{\tilde{f}}_{j}^{(\varepsilon)}$ also have the similar stipulation.

Lemma 3.2. Suppose that $u\left(x, t_{r}\right), v\left(x, t_{r}\right)$ and $w\left(x, t_{r}\right)$ satisfy the $r$-th nonlinear evolution equations (2.16). Moreover, let $P \in \mathscr{K}_{m-1} \backslash\left\{P_{\infty_{1}}, P_{\infty_{2}}\right\},\left(x, x_{0}, t_{r}, t_{0, r}\right) \in \mathbb{C}^{4}$. Then

$$
\psi_{2}\left(P, x, x_{0}, t_{r}, t_{0, r}\right)=\left\{\begin{array}{r}
\exp \left(\zeta^{-1}\left(x-x_{0}\right)+\sum_{l=0}^{r}\left(\tilde{\delta}_{r-l}+\tilde{\beta}_{r-l} \zeta\right) \zeta^{-2 l-2}\left(t_{r}-t_{0, r}\right)\right. \\
\left.+\frac{1}{2} \partial^{-1} u\left(x, t_{r}\right)-\frac{1}{2} \partial^{-1} u\left(x_{0}, t_{0, r}\right)+O(\zeta)\right) \\
\text { as } P \rightarrow P_{\infty_{1}}, \zeta=\lambda^{-1 / 2} \\
\frac{v\left(x, t_{r}\right)}{v\left(x_{0}, t_{0, r}\right)} \exp \left(\sum_{l=0}^{r} \tilde{\alpha}_{r-l} \zeta^{-l}\left(t_{r}-t_{0, r}\right)+O(\zeta)\right) \\
\text { as } P \rightarrow P_{\infty_{2}}, \quad \zeta=\lambda^{-1}
\end{array}\right.
$$

Proof. To investigate the property of $\psi_{2}\left(P, x, x_{0}, t_{r}, t_{0, r}\right)$ near $P_{\infty_{1}}$, one shall take the local coordinate as $\lambda=\zeta^{-2}$. We use the inductive method to prove the subsequent expression

$$
\begin{aligned}
& \bar{I}_{r}^{(1)}\left(P, x, t_{r}\right)=\frac{\partial^{-1}\left[-v w \overline{\tilde{b}}_{r, x}^{(1)}+\left(\partial u-\partial^{2}\right) \overline{\tilde{c}}_{r}^{(1)}+w \overline{\tilde{\tilde{d}}}_{r, x}^{(1)}+(\partial v+v \partial) \overline{\tilde{e}}_{r}^{(1)}\right]}{2}+O(\zeta), \\
& \bar{I}_{r}^{(2)}\left(P, x, t_{r}\right)=\zeta^{-2 r-1}+\frac{\partial^{-1}\left[-v w \tilde{\tilde{b}}_{r, x}^{(2)}+\left(\partial u-\partial^{2}\right) \overline{\tilde{c}}_{r}^{(2)}+w \overline{\tilde{d}}_{r, x}^{(2)}+(\partial v+v \partial) \overline{\tilde{e}}_{r}^{(2)}\right]}{2}+O(\zeta), \\
& \bar{I}_{r}^{(3)}\left(P, x, t_{r}\right)=\zeta^{-2 r-2}+\frac{\partial^{-1}\left[-v w \tilde{\tilde{b}}_{r, x}^{(3)}+\left(\partial u-\partial^{2}\right) \stackrel{\tilde{\tilde{c}}}{r}_{r}^{(3)}+w \overline{\tilde{d}}_{r, x}^{(3)}+(\partial v+v \partial) \overline{\tilde{e}}_{r}^{(3)}\right]}{2}+O(\zeta) .
\end{aligned}
$$

In fact, for $r=1$, a direct calculation shows that

$$
\bar{I}_{1}^{(1)}\left(P, x, t_{r}\right)=\frac{\partial^{-1}\left[-v w \overline{\tilde{b}}_{1, x}^{(1)}+\left(\partial u-\partial^{2}\right) \overline{\tilde{c}}_{1}^{(1)}+w \overline{\tilde{d}}_{1, x}^{(1)}+(\partial v+v \partial) \overline{\tilde{e}}_{1}^{(1)}\right]}{2}+O(\zeta)
$$

Suppose that $\bar{I}_{r}^{(1)}\left(P, x, t_{r}\right)$ has the following expansion

$$
\bar{I}_{r}^{(1)}\left(P, x, t_{r}\right) \underset{\zeta \rightarrow 0}{=} \sum_{j=0}^{\infty} \sigma_{j}^{(1)}\left(x, t_{r}\right) \zeta^{j}, \quad P \rightarrow P_{\infty_{1}}
$$


for some coefficients $\left\{\sigma_{j}^{(1)}\left(x, t_{r}\right)\right\}_{j \in \mathbb{N}_{0}}$ to be determined. Observing

$$
\phi_{2, t_{r}}=\left[\overline{\widetilde{V}}_{21}^{(r, 1)} \phi_{2}+\lambda \overline{\widetilde{V}}_{22}^{(r, 1)}+\frac{\overline{\widetilde{V}}_{23}^{(r, 1)}}{v}\left(\phi_{2, x}+\phi_{2}^{2}-u \phi_{2}-\lambda\right)\right]_{x},
$$

we arrive at

$$
\sigma_{j, x}^{(1)}=\kappa_{1, j, t_{r}}, \quad j=0,1,2,3 \ldots,
$$

Taking use of (2.2), (2.14) and lemma 3.1, we get three expressions

$$
\begin{aligned}
& \sigma_{0}^{(1)}=\partial^{-1} \kappa_{1,0, t_{r}}=-\overline{\tilde{b}}_{r+1}^{(1)} \\
& \sigma_{1}^{(1)}=\partial^{-1} \kappa_{1,1, t_{r}}=\tilde{\tilde{b}}_{r+1}^{(1)}-\overline{\tilde{c}}_{r+1}^{(1)}, \\
& \sigma_{2}^{(1)}=\partial^{-1} \kappa_{1,2, t_{r}}=-\frac{1}{4} \tilde{\tilde{b}}_{r+1, x x}^{(1)}+\frac{1}{4} u \overline{\tilde{b}}_{r+1, x}^{(1)}+\frac{1}{4} v \overline{\tilde{e}}_{r+1}^{(1)}-\frac{3}{4} w \overline{\tilde{d}}_{r+1}^{(1)}+\frac{1}{2} \overline{\tilde{f}}_{r+1}^{(1)},
\end{aligned}
$$

where the integration constants are taken as zero because there is no arbitrary constants in the expansions of $\phi_{2}\left(P, x, t_{r}\right)$ near $P_{\infty_{1}}$ nor in the coefficients of the homogeneous polynomials $\overline{\widetilde{V}}_{i, j}^{(r, 1)}$ with the condition $\partial \partial^{-1}=\partial^{-1} \partial=1$. It is easy to see that

$$
\begin{aligned}
& \bar{I}_{r+1}^{(1)}\left(P, x, t_{r}\right)=\zeta^{-2} \bar{I}_{r}^{(1)}+\left(\overline{\tilde{c}}_{r+1}^{(1)}-\overline{\tilde{b}}_{r+1, x}^{(1)}\right) \phi_{2}+\overline{\tilde{b}}_{r+1}^{(1)} \zeta^{-2}+\frac{\overline{\tilde{d}}_{r+1}^{(1)}}{v}\left(\phi_{2, x}+\phi_{2}^{2}-u \phi_{2}-\zeta^{-2}\right) \\
& =\frac{\partial^{-1}\left[-v w \tilde{\tilde{b}}_{r+1, x}^{(1)}+\left(\partial u-\partial^{2}\right) \overline{\tilde{c}}_{r+1}^{(1)}+w \tilde{\tilde{d}}_{r+1, x}^{(1)}+(\partial v+v \partial) \tilde{\tilde{e}}_{r+1}^{(1)}\right]}{2}+O(\zeta) .
\end{aligned}
$$

Thus $\bar{I}_{r}^{(1)}\left(P, x, t_{r}\right)$ is proved to have the expansion as seen in (3.25) near $P_{\infty_{1}}$. Similarly, one can prove the other two expressions in (3.25), which yield the expansion of $I_{r}\left(P, x, t_{r}\right)$ near $P_{\infty_{1}}$ as follows

$$
I_{r}\left(P, x, t_{r}\right)=\sum_{l=0}^{r}\left(\tilde{\delta}_{r-l}+\tilde{\beta}_{r-l} \zeta\right) \zeta^{-2 l-2}+\frac{\partial^{-1}\left[-v w \tilde{b}_{r, x}+\left(\partial u-\partial^{2}\right) \tilde{c}_{r}+w \tilde{d}_{r, x}+(\partial v+v \partial) \tilde{e}_{r}\right]}{2}+O(\zeta) .
$$

Substituting (3.12) and (3.31) into (3.20), we arrive at the first expression in (3.24) right now. Under the local coordinate $\zeta=\lambda^{-1}$ near $P_{\infty_{2}}$, we can similarly prove the second expression in lemma 3.2.

Let $\omega_{P_{\infty_{s}}, j}^{(2)}(P), j \geq 2, s=1,2$, denote the normalized Abelian differential of the second kind holomorphic on $\mathscr{K}_{m-1} \backslash\left\{P_{\infty_{s}}\right\}$ satisfying

$$
\begin{gathered}
\int_{\mathrm{a}_{k}} \omega_{P_{\infty_{s}}, j}^{(2)}(P)=0, \quad k=1, \ldots, 3 n+1, \\
\omega_{P_{\infty_{s}}, j}^{(2)}(P) \underset{\zeta \rightarrow 0}{=}\left(\zeta^{-j}+O(1)\right) d \zeta, \quad \text { as } \quad P \rightarrow P_{\infty_{s}}, \quad \zeta=\lambda^{1 /(s-3)} .
\end{gathered}
$$

According to the asymptotic behaviors of $\psi_{2}\left(P, x, x_{0}, t_{r}, t_{0, r}\right)$ in (3.24), we introduce the corresponding Abelian differential of the second kind

$$
\Omega_{2}^{(2)}(P)=\omega_{P_{\infty_{1}}, 2}^{(2)}(P)
$$


and

$$
\widetilde{\Omega}_{2 r+3}^{(2)}(P)=\sum_{l=0}^{r}(2 l+2) \tilde{\delta}_{r-l} \omega_{P_{\infty_{1}}, 2 l+3}^{(2)}(P)+\sum_{l=0}^{r}(2 l+1) \tilde{\beta}_{r-l} \omega_{P_{\infty_{1}}, 2 l+2}^{(2)}(P)+\sum_{l=1}^{r} l \tilde{\alpha}_{r-l} \omega_{P_{\infty_{2}}, l+1}^{(2)}(P) .
$$

From (3.33), (3.34) and (3.35), we conclude that

$$
\begin{gathered}
\int_{Q_{0}}^{P} \Omega_{2}^{(2)}(P)=\left\{\begin{array}{l}
-\zeta^{-1}+e_{1}^{(2)}\left(Q_{0}\right)+O(\zeta), \text { as } P \rightarrow P_{\infty_{1}}, \quad \zeta=\lambda^{-1 / 2}, \\
e_{2}^{(2)}\left(Q_{0}\right)+O(\zeta), \text { as } P \rightarrow P_{\infty_{2}}, \quad \zeta=\lambda^{-1},
\end{array}\right. \\
\int_{Q_{0}}^{P} \widetilde{\Omega}_{2 r+3}^{(2)}(P)=\left\{\begin{array}{l}
-\sum_{l=0}^{r} \tilde{\delta}_{r-l} \zeta^{-2 l-2}-\sum_{l=0}^{r} \tilde{\beta}_{r-l} \zeta^{-2 l-1}+\tilde{e}_{1}^{(2)}\left(Q_{0}\right)+O(\zeta), \\
-\sum_{l=1}^{r} \tilde{\alpha}_{r-l} \zeta^{-l}+\tilde{e}_{1}^{(2)}\left(Q_{0}\right)+O(\zeta), \text { as } P \rightarrow P_{\infty_{1}}, \quad \zeta=\lambda_{\infty_{2}}, \quad \zeta=\lambda^{-1 / 2},
\end{array}\right.
\end{gathered}
$$

where $e_{1}^{(2)}\left(Q_{0}\right), e_{2}^{(2)}\left(Q_{0}\right), \tilde{e}_{1}^{(2)}\left(Q_{0}\right)$ and $\tilde{e}_{2}^{(2)}\left(Q_{0}\right)$ are integration constants with $Q_{0}$ an appropriately chosen base point on $\mathscr{K}_{m-1} \backslash\left\{P_{\infty_{1}}, P_{\infty_{2}}\right\}$. The b -periods of the differential $\Omega_{2}^{(2)}(P)$ and $\widetilde{\Omega}_{2 r+3}^{(2)}(P)$ are denoted by

$$
\begin{gathered}
\underline{U}_{2}^{(2)}=\left(U_{2,1}^{(2)}, \ldots, U_{2, m-1}^{(2)}\right), \quad U_{2, k}^{(2)}=\frac{1}{2 \pi i} \int_{\mathbb{b}_{k}} \Omega_{2}^{(2)}(P), \quad k=1, \ldots, m-1, \\
\underline{\widetilde{U}}_{2 r+3}^{(2)}=\left(\widetilde{U}_{2 r+3,1}^{(2)}, \ldots, \widetilde{U}_{2 r+3, m-1}^{(2)}\right), \quad \widetilde{U}_{2 r+3, j}^{(2)}=\frac{1}{2 \pi i} \int_{\mathbb{b}_{j}} \widetilde{\Omega}_{2 r+3}^{(2)}(P), \quad j=1, \ldots, m-1 .
\end{gathered}
$$

By the relationship between the normalized Abelian differential of the second kind and the normalized holomorphic differential $\underline{\omega}$, we can derive that

$$
\begin{gathered}
U_{2, k}^{(2)}=\rho_{k, 0}\left(P_{\infty_{1}}\right), \quad k=1,2, \ldots, m-1, \\
\widetilde{U}_{2 r+3, k}^{(2)}=\sum_{l=0}^{r} \tilde{\delta}_{r-l} \rho_{k, 2 l+1}\left(P_{\infty_{1}}\right)+\sum_{l=0}^{r} \tilde{\beta}_{r-l} \rho_{k, 2 l}\left(P_{\infty_{1}}\right)+\sum_{l=1}^{r} \tilde{\alpha}_{r-l} \rho_{k, l-1}\left(P_{\infty_{2}}\right), \quad k=1,2, \ldots, m-1 .
\end{gathered}
$$

\section{Divisors of meromorphic functions and Baker-Akhiezer function}

In this section, we shall investigate the properties of meromorphic functions and Baker-Akhiezer function on the finite part of the Riemann surface $\mathscr{K}_{m-1}$ including the divisors, which are necessary for construction of the Riemann theta function representations.

For convenience, we define three points $P, P^{*}, P^{* *}$ on three different sheets of the same Riemann surface $\mathscr{K}_{m-2}$. For a fixed $\lambda$, let $y_{i}(\lambda), i=0,1,2$, denote the three roots of polynomial $\mathscr{F}_{m}(\lambda, y)=0$, that is

$$
\left(y-y_{0}(\lambda)\right)\left(y-y_{1}(\lambda)\right)\left(y-y_{2}(\lambda)\right)=y^{3}-y^{2} R_{m}+y S_{m}-T_{m}=0 .
$$

Then points $\left(\lambda, y_{0}(\lambda)\right),\left(\lambda, y_{1}(\lambda)\right)$ and $\left(\lambda, y_{2}(\lambda)\right)$ are on the three different sheets of Riemann surface $\mathscr{K}_{m-1}$, respectively. Let $P=\left(\lambda, y_{i}(\lambda)\right), i=0,1,2$, be an arbitrary point in the three points, 
then the other two points are defined as $P^{*}$ and $P^{* *}$, respectively. From (4.1), we can derive the relationships between the roots $y_{i}(\lambda), i=0,1,2$ and the coefficients $R_{m}, S_{m}, T_{m}$

$$
\begin{aligned}
& y_{0}+y_{1}+y_{2}=R_{m}, \\
& y_{0} y_{1}+y_{0} y_{2}+y_{1} y_{2}=S_{m}, \\
& y_{0} y_{1} y_{2}=T_{m}, \\
& y_{0}^{2}+y_{1}^{2}+y_{2}^{2}=R_{m}^{2}-2 S_{m}, \\
& y_{0}^{3}+y_{1}^{3}+y_{2}^{3}=R_{m}^{3}-3 R_{m} S_{m}+3 T_{m}, \\
& y_{0}^{2} y_{1}^{2}+y_{0}^{2} y_{2}^{2}+y_{1}^{2} y_{2}^{2}=S_{m}^{2}-2 R_{m} T_{m}, \\
& \prod_{j=0}^{2}\left(3 y_{j}^{2}-2 y_{j} R_{m}+S_{m}\right)=\Delta(\lambda) .
\end{aligned}
$$

Using (3.9), (3.10) and (3.11), a direct calculation shows that

$$
\begin{gathered}
\phi_{2}=\frac{y V_{13}^{(n)}+C_{m}}{y V_{23}^{(n)}+A_{m}}=\frac{\lambda F_{m-1}}{y^{2} V_{13}^{(n)}-y\left(R_{m} V_{13}^{(n)}+C_{m}\right)+D_{m}}=\frac{y^{2} V_{23}^{(n)}-y\left(R_{m} V_{23}^{(n)}+A_{m}\right)+B_{m}}{E_{m-1}}, \\
\phi_{3}=\frac{y V_{12}^{(n)}+\mathscr{C}_{m}}{y V_{32}^{(n)}+\mathscr{A}_{m}}=\frac{-F_{m-1}}{y^{2} V_{12}^{(n)}-y\left(R_{m} V_{12}^{(n)}+\mathscr{C}_{m}\right)+\mathscr{D}_{m}}=\frac{y^{2} V_{32}^{(n)}-y\left(R_{m} V_{32}^{(n)}+\mathscr{A}_{m}\right)+\mathscr{B}_{m}}{\mathscr{E}_{m-1}},
\end{gathered}
$$

where

$$
\begin{aligned}
& A_{m}=V_{13}^{(n)} V_{21}^{(n)}-V_{11}^{(n)} V_{23}^{(n)}, \\
& B_{m}=\lambda\left(V_{22}^{(n)} V_{23}^{(n)} V_{33}^{(n)}-\left(V_{23}^{(n)}\right)^{2} V_{32}^{(n)}+V_{13}^{(n)} V_{21}^{(n)} V_{22}^{(n)}-V_{12}^{(n)} V_{21}^{(n)} V_{23}^{(n)}\right), \\
& C_{m}=\lambda\left(V_{12}^{(n)} V_{23}^{(n)}-V_{13}^{(n)} V_{22}^{(n)}\right), \\
& D_{m}=V_{11}^{(n)} V_{13}^{(n)} V_{33}^{(n)}-\left(V_{13}^{(n)}\right)^{2} V_{31}^{(n)}+\lambda V_{11}^{(n)} V_{12}^{(n)} V_{23}^{(n)}-\lambda V_{12}^{(n)} V_{13}^{(n)} V_{21}^{(n)}, \\
& \mathscr{A}_{m}=V_{12}^{(n)} V_{31}^{(n)}-V_{11}^{(n)} V_{32}^{(n)}, \\
& \mathscr{B}_{m}=\lambda V_{22}^{(n)} V_{32}^{(n)} V_{33}^{(n)}-\lambda V_{23}^{(n)}\left(V_{32}^{(n)}\right)^{2}+V_{12}^{(n)} V_{31}^{(n)} V_{33}^{(n)}-V_{13}^{(n)} V_{31}^{(n)} V_{32}^{(n)}, \\
& \mathscr{C}_{m}=V_{13}^{(n)} V_{32}^{(n)}-V_{12}^{(n)} V_{33}^{(n)}, \\
& \mathscr{D}_{m}=\lambda V_{11}^{(n)} V_{12}^{(n)} V_{22}^{(n)}-\lambda\left(V_{12}^{(n)}\right)^{2} V_{21}^{(n)}+V_{11}^{(n)} V_{13}^{(n)} V_{32}^{(n)}-V_{12}^{(n)} V_{13}^{(n)} V_{31}^{(n)}, \\
& E_{m-1}=V_{21}^{(n)}\left(V_{11}^{(n)} V_{23}^{(n)}-V_{13}^{(n)} V_{21}^{(n)}\right)+V_{23}^{(n)}\left(V_{23}^{(n)} V_{31}^{(n)}-V_{21}^{(n)} V_{33}^{(n)}\right), \\
& F_{m-1}=V_{13}^{(n)}\left(V_{13}^{(n)} V_{32}^{(n)}-V_{12}^{(n)} V_{33}^{(n)}\right)+\lambda V_{12}^{(n)}\left(V_{13}^{(n)} V_{22}^{(n)}-V_{12}^{(n)} V_{23}^{(n)}\right), \\
& \mathscr{E}_{m-1}=V_{31}^{(n)}\left(V_{11}^{(n)} V_{32}^{(n)}-V_{12}^{(n)} V_{31}^{(n)}\right)+\lambda V_{32}^{(n)}\left(V_{21}^{(n)} V_{32}^{(n)}-V_{22}^{(n)} V_{31}^{(n)}\right) .
\end{aligned}
$$

It can be inferred from (4.7) that $E_{m-1}, F_{m-1}$ and $\mathscr{E}_{m-1}$ are polynomials with respect to $\lambda$ of degree $3 n+1$ for $\beta_{0} \delta_{0} \neq 0$. Therefore, we can rewrite them in the following form:

$$
\begin{aligned}
& E_{m-1}\left(\lambda, x, t_{r}\right)=\beta_{0} \delta_{0}^{2} v \prod_{j=1}^{3 n+1}\left(\lambda-\mu_{j}\left(x, t_{r}\right)\right), \\
& F_{m-1}\left(\lambda, x, t_{r}\right)=\beta_{0} \delta_{0}^{2} v_{x} \prod_{j=1}^{3 n+1}\left(\lambda-v_{j}\left(x, t_{r}\right)\right),
\end{aligned}
$$




$$
\mathscr{E}_{m-1}\left(\lambda, x, t_{r}\right)=\beta_{0} \delta_{0}^{2} w^{2} \prod_{j=1}^{3 n+1}\left(\lambda-\xi_{j}\left(x, t_{r}\right)\right),
$$

where $\left\{\mu_{j}\left(x, t_{r}\right)\right\}_{j=1}^{3 n+1},\left\{v_{j}\left(x, t_{r}\right)\right\}_{j=1}^{3 n+1},\left\{\xi_{j}\left(x, t_{r}\right)\right\}_{j=1}^{3 n+1}$ are zeros of $E_{m-1}\left(\lambda, x, t_{r}\right), F_{m-1}\left(\lambda, x, t_{r}\right)$, $\mathscr{E}_{m-1}\left(\lambda, x, t_{r}\right)$ respectively. Define $P_{0}=(0,0)$. Since

$$
\frac{\mathscr{C}_{m}\left(v_{j}\left(x, t_{r}\right), x, t_{r}\right)}{V_{12}^{(n)}\left(v_{j}\left(x, t_{r}\right), x, t_{r}\right)}=\frac{C_{m}\left(v_{j}\left(x, t_{r}\right), x, t_{r}\right)}{V_{13}^{(n)}\left(v_{j}\left(x, t_{r}\right), x, t_{r}\right)}
$$

which can be deduced from

$$
\begin{aligned}
& \left.F_{m-1}\right|_{\lambda=v_{j}\left(x, t_{r}\right)}=\left.\left[V_{13}^{(n)}\left(V_{13}^{(n)} V_{32}^{(n)}-V_{12}^{(n)} V_{33}^{(n)}\right)-\lambda V_{12}^{(n)}\left(V_{12}^{(n)} V_{23}^{(n)}-V_{13}^{(n)} V_{22}^{(n)}\right)\right]\right|_{\lambda=v_{j}\left(x, t_{r}\right)} \\
& =\left[V_{13}^{(n)}\left(v_{j}\left(x, t_{r}\right), x, t_{r}\right) \mathscr{C}_{m}\left(v_{j}\left(x, t_{r}\right), x, t_{r}\right)-V_{12}^{(n)}\left(v_{j}\left(x, t_{r}\right), x, t_{r}\right) C_{m}\left(v_{j}\left(x, t_{r}\right), x, t_{r}\right)\right]=0,
\end{aligned}
$$

we can define

$$
\begin{aligned}
\hat{\mu}_{j}\left(x, t_{r}\right)=\left(\mu_{j}\left(x, t_{r}\right), y\left(\mu_{j}\left(x, t_{r}\right)\right)\right) & =\left(\mu_{j}\left(x, t_{r}\right),-\frac{A_{m}\left(\mu_{j}\left(x, t_{r}\right), x, t_{r}\right)}{V_{23}^{(n)}\left(\mu_{j}\left(x, t_{r}\right), x, t_{r}\right)}\right) \in \mathscr{K}_{m-1}, \\
\hat{v}_{j}\left(x, t_{r}\right)=\left(v_{j}\left(x, t_{r}\right), y\left(v_{j}\left(x, t_{r}\right)\right)\right) & =\left(v_{j}\left(x, t_{r}\right),-\frac{C_{m}\left(v_{j}\left(x, t_{r}\right), x, t_{r}\right)}{V_{13}^{(n)}\left(v_{j}\left(x, t_{r}\right), x, t_{r}\right)}\right) \\
& =\left(v_{j}\left(x, t_{r}\right),-\frac{\mathscr{C}_{m}\left(v_{j}\left(x, t_{r}\right), x, t_{r}\right)}{V_{12}^{(n)}\left(v_{j}\left(x, t_{r}\right), x, t_{r}\right)}\right) \in \mathscr{K}_{m-1}, \\
\hat{\xi}_{j}\left(x, t_{r}\right)=\left(\xi_{j}\left(x, t_{r}\right), y\left(\xi_{j}\left(x, t_{r}\right)\right)\right) & =\left(\xi_{j}\left(x, t_{r}\right),-\frac{\mathscr{A}_{m}\left(\xi_{j}\left(x, t_{r}\right), x, t_{r}\right)}{V_{32}^{(n)}\left(\xi_{j}\left(x, t_{r}\right), x, t_{r}\right)}\right) \in \mathscr{K}_{m-1}
\end{aligned}
$$

with $1 \leq j \leq 3 n+1,\left(x, t_{r}\right) \in \mathbb{C}^{2}$.

Observing (4.3), (4.4) and lemma 3.1, we obtain the divisors $\left(\phi_{2}\left(P, x, t_{r}\right)\right)$ and $\left(\phi_{3}\left(P, x, t_{r}\right)\right)$ of $\phi_{2}\left(P, x, t_{r}\right)$ and $\phi_{3}\left(P, x, t_{r}\right)$ as follows

$$
\begin{aligned}
& \left(\phi_{2}\left(P, x, t_{r}\right)\right)=\mathscr{D}_{P_{0}, \hat{v}_{1}\left(x, t_{r}\right), \ldots, \hat{v}_{3 n+1}\left(x, t_{r}\right)}(P)-\mathscr{D}_{P_{\infty_{1}}, \hat{\mu}_{1}\left(x, t_{r}\right), \ldots, \hat{\mu}_{3 n+1}\left(x, t_{r}\right)}(P), \\
& \left(\phi_{3}\left(P, x, t_{r}\right)\right)=\mathscr{D}_{P_{\infty_{2}}, \hat{v}_{1}\left(x, t_{r}\right), \ldots, \hat{v}_{3 n+1}\left(x, t_{r}\right)}(P)-\mathscr{D}_{P_{\infty_{1}}, \hat{\xi}_{1}\left(x, t_{r}\right), \ldots, \hat{\xi}_{3 n+1}\left(x, t_{r}\right)}(P) .
\end{aligned}
$$

Now we are in a position to discuss zeros and poles of $\psi_{2}\left(P, x, x_{0}, t_{r}, t_{0, r}\right)$ on $\mathscr{K}_{m-1} \backslash$ $\left\{P_{\infty_{1}}, P_{\infty_{2}}\right\}$. From (4.3) and (4.4), we can easily obtain the interrelationships among the polynomials $A_{m}, B_{m}, C_{m}, D_{m}, \mathscr{A}_{m}, \mathscr{B}_{m}, \mathscr{C}_{m}, \mathscr{D}_{m}, E_{m-1}, F_{m-1}, \mathscr{E}_{m-1}, R_{m}, S_{m}, T_{m}$, which we list below:

$$
\begin{aligned}
& \lambda V_{23}^{(n)} F_{m-1}=V_{13}^{(n)} D_{m}-\left(V_{13}^{(n)}\right)^{2} S_{m}-V_{13}^{(n)} R_{m} C_{m}-C_{m}^{2}, \\
& \lambda A_{m} F_{m-1}=\left(V_{13}^{(n)}\right)^{2} T_{m}+C_{m} D_{m}, \\
& V_{13}^{(n)} E_{m-1}=V_{23}^{(n)} B_{m}-\left(V_{23}^{(n)}\right)^{2} S_{m}-V_{23}^{(n)} R_{m} A_{m}-A_{m}^{2}, \\
& C_{m} E_{m-1}=\left(V_{23}^{(n)}\right)^{2} T_{m}+A_{m} B_{m},
\end{aligned}
$$




$$
\begin{aligned}
& 0=V_{13}^{(n)} B_{m}+V_{23}^{(n)} D_{m}-V_{13}^{(n)} V_{23}^{(n)} S_{m}+A_{m} C_{m}, \\
& 0=V_{13}^{(n)} V_{23}^{(n)} R_{m} S_{m}+V_{13}^{(n)} V_{23}^{(n)} T_{m}+V_{13}^{(n)} A_{m} S_{m}+V_{23}^{(n)} C_{m} S_{m}-V_{13}^{(n)} R_{m} B_{m}-V_{23}^{(n)} R_{m} D_{m}-B_{m} C_{m}-A_{m} D_{m}, \\
& 0=V_{13}^{(n)} V_{23}^{(n)} R_{m} T_{m}+V_{13}^{(n)} A_{m} T_{m}+V_{23}^{(n)} C_{m} T_{m}+\lambda E_{m-1} F_{m-1}-B_{m} D_{m}, \\
& -V_{32}^{(n)} F_{m-1}=V_{12}^{(n)} \mathscr{D}_{m}-\left(V_{12}^{(n)}\right)^{2} S_{m}-V_{12}^{(n)} R_{m} \mathscr{C}_{m}-\mathscr{C}_{m}^{2}, \\
& -\mathscr{A}_{m} F_{m-1}=\left(V_{12}^{(n)}\right)^{2} T_{m}+\mathscr{C}_{m} \mathscr{D}_{m}, \\
& V_{12}^{(n)} \mathscr{E}_{m-1}=V_{32}^{(n)} \mathscr{B}_{m}-\left(V_{32}^{(n)}\right)^{2} S_{m}-V_{32}^{(n)} R_{m} \mathscr{A}_{m}-\mathscr{A}_{m}^{2}, \\
& \mathscr{C}_{m} \mathscr{E}_{m-1}=\left(V_{32}^{(n)}\right)^{2} T_{m}+\mathscr{A}_{m} \mathscr{B}_{m}, \\
& 0=V_{12}^{(n)} \mathscr{B}_{m}+V_{32}^{(n)} \mathscr{D}_{m}-V_{12}^{(n)} V_{32}^{(n)} S_{m}+\mathscr{A}_{m} \mathscr{C}_{m}, \\
& 0=V_{12}^{(n)} V_{32}^{(n)} R_{m} S_{m}+V_{12}^{(n)} V_{32}^{(n)} T_{m}+V_{12}^{(n)} \mathscr{A}_{m} S_{m}+V_{32}^{(n)} \mathscr{C}_{m} S_{m}-V_{12}^{(n)} R_{m} \mathscr{B}_{m}-V_{32}^{(n)} R_{m} \mathscr{D}_{m}-\mathscr{B}_{m} \mathscr{C}_{m}-\mathscr{A}_{m} \mathscr{D}_{m}, \\
& 0=V_{12}^{(n)} V_{32}^{(n)} R_{m} T_{m}+V_{12}^{(n)} \mathscr{A}_{m} T_{m}+V_{32}^{(n)} \mathscr{C}_{m} T_{m}-\mathscr{E}_{m-1} F_{m-1}-\mathscr{B}_{m} \mathscr{D}_{m} .
\end{aligned}
$$

Taking use of above relationships, we arrive at the evolution of $E_{m-1}\left(\lambda, x, t_{r}\right), F_{m-1}\left(\lambda, x, t_{r}\right)$, $\mathscr{E}_{m-1}\left(\lambda, x, t_{r}\right)$ with respect to $x$ and $t_{r}$ respectively in the subsequent lemma.

Lemma 4.1. Assume that (3.9) holds and let $\left(\lambda, x, t_{r}\right) \in \mathbb{C}^{3}$. Then

$$
\begin{aligned}
E_{m-1, x}=-u E_{m-1}- & \left(R_{m} A_{m}+2 V_{23}^{(n)} S_{m}-3 B_{m}\right) \\
F_{m-1, x}=2 u F_{m-1} & +\left(-R_{m} C_{m}-2 V_{13}^{(n)} S_{m}+3 D_{m}\right)+v\left(R_{m} \mathscr{C}_{m}+2 V_{12}^{(n)} S_{m}-3 \mathscr{D}_{m}\right) \\
\mathscr{E}_{m-1, x}=-u \mathscr{E}_{m-1}+w\left(-R_{m} \mathscr{A}_{m}-2 V_{32}^{(n)} S_{m}+3 \mathscr{B}_{m}\right) & \\
E_{m-1, t_{r}}\left(\lambda, x, t_{r}\right)= & E_{m-1}\left[-\partial^{-1} u_{t_{r}}+3 \lambda \widetilde{V}_{22}^{(r)}+\frac{\widetilde{V}_{23}^{(r)}\left(R_{m}-3 \lambda V_{22}^{(n)}\right)}{V_{23}^{(n)}}\right] \\
+ & \frac{\widetilde{V}_{23}^{(r)} V_{21}^{(n)}-\widetilde{V}_{21}^{(r)} V_{23}^{(n)}}{V_{23}^{(n)}\left(R_{m} A_{m}+2 V_{23}^{(n)} S_{m}-3 B_{m}\right)} \\
= & E_{m-1}\left[-\partial^{-1} u_{t_{r}}+3 \lambda \widetilde{V}_{22}^{(r)}+u \widetilde{V}_{21}^{(r)}+\frac{\widetilde{V}_{23}^{(r)}\left(R_{m}-u V_{21}^{(n)}-3 \lambda V_{22}^{(n)}\right)}{V_{23}^{(n)}}\right] \\
+ & \frac{\widetilde{V}_{21}^{(r)} V_{23}^{(n)}-\widetilde{V}_{23}^{(r)} V_{21}^{(n)} E_{m-1, x},}{V_{23}^{(n)}} \\
F_{m-1, t_{r}}\left(\lambda, x, t_{r}\right)= & \left(3 \widetilde{V}_{11}^{(r)}-\partial^{-1} u_{t_{r}}\right) F_{m-1}+\widetilde{V}_{21}^{(r)}\left(-R_{m} C_{m}-2 V_{13}^{(n)} S_{m}+3 D_{m}\right) \\
& +\widetilde{V}_{13}^{(r)}\left(R_{m} \mathscr{C}_{m}+2 V_{12}^{(n)} S_{m}-3 \mathscr{D}_{m}\right) \\
= & F_{m-1}\left[3 \widetilde{V}_{11}^{(r)}-\partial^{-1} u_{t_{r}}+\frac{\widetilde{V}_{12}^{(r)}\left(v R_{m}-3 v V_{11}^{(n)}+2 u V_{13}^{(n)}\right)}{v V_{12}^{(n)}-V_{13}^{(n)}}\right. \\
& \left.\quad \frac{\widetilde{V}_{13}^{(r)}\left(R_{m}-3 V_{11}^{(n)}+2 u V_{12}^{(n)}\right)}{v V_{12}^{(n)}-V_{13}^{(n)}}\right]-\frac{\widetilde{V}_{12}^{(r)} V_{13}^{(n)}-V_{12}^{(n)} \widetilde{V}_{13}^{(r)}}{v V_{12}^{(n)}-V_{13}^{(n)}} F_{m-1, x}
\end{aligned}
$$




$$
\begin{aligned}
\mathscr{E}_{m-1, t_{r}}\left(\lambda, x, t_{r}\right)= & \mathscr{E}_{m-1}\left[-\partial^{-1} u_{t_{r}}+3 \widetilde{V}_{33}^{(r)}+\frac{u \widetilde{V}_{31}^{(r)}}{w}+\frac{\widetilde{V}_{32}^{(r)}}{w V_{32}^{(n)}}\left(w R_{m}-u V_{31}^{(n)}-3 w V_{33}^{(n)}\right)\right] \\
& +\frac{\widetilde{V}_{31}^{(r)} V_{32}^{(n)}-V_{31}^{(n)} \widetilde{V}_{32}^{(r)}}{w V_{32}^{(n)}} \mathscr{E}_{m-1, x} \\
= & \mathscr{E}_{m-1}\left[-\partial^{-1} u_{t_{r}}+3 \widetilde{V}_{33}^{(r)}+\frac{\widetilde{V}_{32}^{(r)}\left(R_{m}-3 V_{33}^{(n)}\right)}{V_{32}^{(n)}}\right] \\
& +\frac{V_{31}^{(n)} \widetilde{V}_{32}^{(r)}-\widetilde{V}_{31}^{(r)} V_{32}^{(n)}}{V_{32}^{(n)}}\left(R_{m} \mathscr{A}_{m}+2 V_{32}^{(n)} S_{m}-3 \mathscr{B}_{m}\right)
\end{aligned}
$$

Proof. Considering that $V^{(n)}$ satisfies the $n$th stationary equation, we can prove equations (4.22) directly. In order to prove (4.23), (4.24) and (4.25), we first show several expressions about the meromorphic functions $\phi_{2}\left(P, x, t_{r}\right)$ and $\phi_{3}\left(P, x, t_{r}\right)$

$$
\begin{aligned}
& \phi_{2}\left(P, x, t_{r}\right)+\phi_{2}\left(P^{*}, x, t_{r}\right)+\phi_{2}\left(P^{* *}, x, t_{r}\right)=\frac{-R_{m} A_{m}-2 V_{23}^{(n)} S_{m}+3 B_{m}}{E_{m-1}\left(\lambda, x, t_{r}\right)}, \\
& \phi_{3}\left(P, x, t_{r}\right)+\phi_{3}\left(P^{*}, x, t_{r}\right)+\phi_{3}\left(P^{* *}, x, t_{r}\right)=\frac{-R_{m} \mathscr{A}_{m}-2 V_{32}^{(n)} S_{m}+3 \mathscr{B}_{m}}{\mathscr{E}_{m-1}\left(\lambda, x, t_{r}\right)}, \\
& \phi_{2}\left(P, x, t_{r}\right) \phi_{2}\left(P^{*}, x, t_{r}\right) \phi_{2}\left(P^{* *}, x, t_{r}\right)=-\frac{\lambda F_{m-1}\left(\lambda, x, t_{r}\right)}{E_{m-1}\left(\lambda, x, t_{r}\right)} \\
& \phi_{3}\left(P, x, t_{r}\right) \phi_{3}\left(P^{*}, x, t_{r}\right) \phi_{3}\left(P^{* *}, x, t_{r}\right)=\frac{F_{m-1}\left(\lambda, x, t_{r}\right)}{\mathscr{E}_{m-1}\left(\lambda, x, t_{r}\right)} \\
& \frac{1}{\phi_{2}\left(P, x, t_{r}\right)}+\frac{1}{\phi_{2}\left(P^{*}, x, t_{r}\right)}+\frac{1}{\phi_{2}\left(P^{* *}, x, t_{r}\right)}=\frac{-R_{m} C_{m}-2 V_{13}^{(n)} S_{m}+3 D_{m}}{\lambda F_{m-1}\left(\lambda, x, t_{r}\right)} \\
& =\frac{\left(v R_{m}-3 v V_{11}^{(n)}+2 u V_{13}^{(n)}\right) F_{m-1}\left(\lambda, x, t_{r}\right)-V_{13}^{(n)} F_{m-1, x}\left(\lambda, x, t_{r}\right)}{\lambda\left(v V_{12}^{(n)}-V_{13}^{(n)}\right) F_{m-1}\left(\lambda, x, t_{r}\right)}, \\
& \frac{1}{\phi_{3}\left(P, x, t_{r}\right)}+\frac{1}{\phi_{3}\left(P^{*}, x, t_{r}\right)}+\frac{1}{\phi_{3}\left(P^{* *}, x, t_{r}\right)}=\frac{R_{m} \mathscr{C}_{m}+2 V_{12}^{(n)} S_{m}-3 \mathscr{D}_{m}}{F_{m-1}\left(\lambda, x, t_{r}\right)} \\
& =\frac{\left(R_{m}-3 V_{11}^{(n)}+2 u V_{12}^{(n)}\right) F_{m-1}\left(\lambda, x, t_{r}\right)-V_{12}^{(n)} F_{m-1, x}\left(\lambda, x, t_{r}\right)}{\left(V_{13}^{(n)}-v V_{12}^{(n)}\right) F_{m-1}\left(\lambda, x, t_{r}\right)}, \\
& \frac{\phi_{2}\left(P, x, t_{r}\right)}{\phi_{3}\left(P, x, t_{r}\right)}+\frac{\phi_{2}\left(P^{*}, x, t_{r}\right)}{\phi_{3}\left(P^{*}, x, t_{r}\right)}+\frac{\phi_{2}\left(P^{* *}, x, t_{r}\right)}{\phi_{3}\left(P^{* *}, x, t_{r}\right)} \\
& =\frac{-V_{21}^{(n)} E_{m-1, x}\left(\lambda, x, t_{r}\right)+\left(R_{m}-u V_{21}^{(n)}-3 \lambda V_{22}^{(n)}\right) E_{m-1}\left(\lambda, x, t_{r}\right)}{V_{23}^{(n)} E_{m-1}\left(\lambda, x, t_{r}\right)},
\end{aligned}
$$




$$
\begin{aligned}
& \frac{\phi_{3}\left(P, x, t_{r}\right)}{\phi_{2}\left(P, x, t_{r}\right)}+\frac{\phi_{3}\left(P^{*}, x, t_{r}\right)}{\phi_{2}\left(P^{*}, x, t_{r}\right)}+\frac{\phi_{3}\left(P^{* *}, x, t_{r}\right)}{\phi_{2}\left(P^{* *}, x, t_{r}\right)} \\
& =\frac{-V_{31}^{(n)} \mathscr{E}_{m-1, x}\left(\lambda, x, t_{r}\right)+\left(w R_{m}-u V_{31}^{(n)}-3 w V_{33}^{(n)}\right) \mathscr{E}_{m-1}\left(\lambda, x, t_{r}\right)}{\lambda w V_{32}^{(n)} \mathscr{E}_{m-1}\left(\lambda, x, t_{r}\right)} .
\end{aligned}
$$

Expression (4.26) implies that

$$
\frac{E_{m-1, x}}{E_{m-1}}=-u+\phi_{2}\left(P, x, t_{r}\right)+\phi_{2}\left(P^{*}, x, t_{r}\right)+\phi_{2}\left(P^{* *}, x, t_{r}\right) .
$$

Differentiating (4.34) with respect to $t_{r}$, we can derive

$$
\begin{aligned}
& \left(\frac{E_{m-1, x}}{E_{m-1}}\right)_{t_{r}}=\partial_{x} \partial_{t_{r}}\left(\ln E_{m-1}\right) \\
= & {\left[-u+\phi_{2}\left(P, x, t_{r}\right)+\phi_{2}\left(P^{*}, x, t_{r}\right)+\phi_{2}\left(P^{* *}, x, t_{r}\right)\right]_{t_{r}} } \\
= & -u_{t_{r}}+\partial_{x}\left(\frac{\psi_{2, t_{r}}\left(P, x, x_{0}, t_{r}, t_{0, r}\right)}{\psi_{2}\left(P, x, x_{0}, t_{r}, t_{0, r}\right)}+\frac{\psi_{2, t_{r}}\left(P^{*}, x, x_{0}, t_{r}, t_{0, r}\right)}{\psi_{2}\left(P^{*}, x, x_{0}, t_{r}, t_{0, r}\right)}+\frac{\psi_{2, t_{r}}\left(P^{* *}, x, x_{0}, t_{r}, t_{0, r}\right)}{\psi_{2}\left(P^{* *}, x, x_{0}, t_{r}, t_{0, r}\right)}\right) \\
= & -u_{t_{r}}+\partial_{x}\left(\widetilde{V}_{21}^{(r)}\left(\phi_{2}\left(P, x, t_{r}\right)+\phi_{2}\left(P^{*}, x, t_{r}\right)+\phi_{2}\left(P^{* *}, x, t_{r}\right)\right)\right. \\
& \left.+3 \lambda \widetilde{V}_{22}^{(r)}+\widetilde{V}_{23}^{(r)}\left(\frac{\phi_{2}\left(P, x, t_{r}\right)}{\phi_{3}\left(P, x, t_{r}\right)}+\frac{\phi_{2}\left(P^{*}, x, t_{r}\right)}{\phi_{3}\left(P^{*}, x, t_{r}\right)}+\frac{\phi_{2}\left(P^{* *}, x, t_{r}\right)}{\phi_{3}\left(P^{* *}, x, t_{r}\right)}\right)\right) .
\end{aligned}
$$

Without loss of generality, taking the integration constant of (4.35) to be zero and substituting (4.26) and (4.32) into (4.35) can indicate (4.23). Expressions (4.24) and (4.25) can be proved similarly.

Lemma 4.1 naturally yields the dynamics of the zeros $\mu_{j}\left(x, t_{r}\right), v_{j}\left(x, t_{r}\right)$ and $\xi_{j}\left(x, t_{r}\right)$ of $E_{m-1}\left(\lambda, x, t_{r}\right), F_{m-1}\left(\lambda, x, t_{r}\right)$ and $\mathscr{E}_{m-1}\left(\lambda, x, t_{r}\right)$ in terms of Dubrovin-type equations in the subsequent lemma.

Lemma 4.2. Suppose that the zeros $\left\{\mu_{j}\left(x, t_{r}\right)\right\}_{j=1, \ldots, 3 n+1}, \quad\left\{v_{j}\left(x, t_{r}\right)\right\}_{j=1, \ldots, 3 n+1}$ and $\left\{\xi_{j}\left(x, t_{r}\right)\right\}_{j=1, \ldots, 3 n+1}$ of $E_{m-1}\left(\lambda, x, t_{r}\right), F_{m-1}\left(\lambda, x, t_{r}\right)$ and $\mathscr{E}_{m-1}\left(\lambda, x, t_{r}\right)$ remain distinct for $\left(x, t_{r}\right) \in$ $\Omega_{\mu},\left(x, t_{r}\right) \in \Omega_{v}$ and $\left(x, t_{r}\right) \in \Omega_{\xi}$, respectively, where $\Omega_{\mu}, \Omega_{v}, \Omega_{\xi} \subseteq \mathbb{C}^{2}$ are open and connected. Then $\left\{\mu_{j}\left(x, t_{r}\right)\right\}_{j=1, \ldots, 3 n+1},\left\{v_{j}\left(x, t_{r}\right)\right\}_{j=1, \ldots, 3 n+1}$ and $\left\{\xi_{j}\left(x, t_{r}\right)\right\}_{j=1, \ldots, 3 n+1}$ satisfy the system of differential equations

$$
\begin{gathered}
\mu_{j, x}\left(x, t_{r}\right)=\frac{-\left.V_{23}^{(n)}\left(3 y^{2}-2 R_{m} y+S_{m}\right)\right|_{\lambda=\mu_{j}\left(x, t_{r}\right)}}{\beta_{0} \delta_{0}^{2} v \prod_{\substack{k=1 \\
k \neq j}}^{3 n+1}\left(\mu_{j}\left(x, t_{r}\right)-\mu_{k}\left(x, t_{r}\right)\right)} \\
\mu_{j, t_{r}}\left(x, t_{r}\right)=\frac{\left.\left(V_{21}^{(n)} \widetilde{V}_{23}^{(r)}-\widetilde{V}_{21}^{(r)} V_{23}^{(n)}\right)\left(3 y^{2}-2 R_{m} y+S_{m}\right)\right|_{\lambda=\mu_{j}\left(x, t_{r}\right)}}{\beta_{0} \delta_{0}^{2} v \prod_{\substack{k=1 \\
k \neq j}}^{3 n+1}\left(\mu_{j}\left(x, t_{r}\right)-\mu_{k}\left(x, t_{r}\right)\right)}, \\
v_{j, x}\left(x, t_{r}\right)=\frac{\left.\left(v V_{12}^{(n)}-V_{13}^{(n)}\right)\left(3 y^{2}-2 R_{m} y+S_{m}\right)\right|_{\lambda=v_{j}\left(x, t_{r}\right)}}{\beta_{0} \delta_{0}^{2} v_{x} \prod_{\substack{k=1 \\
k \neq j}}^{3 n+1}\left(v_{j}\left(x, t_{r}\right)-v_{k}\left(x, t_{r}\right)\right)}
\end{gathered}
$$




$$
\begin{gathered}
v_{j, t_{r}}\left(x, t_{r}\right)=\frac{\left.\left(V_{12}^{(n)} \widetilde{V}_{13}^{(r)}-\widetilde{V}_{12}^{(r)} V_{13}^{(n)}\right)\left(3 y^{2}-2 R_{m} y+S_{m}\right)\right|_{\lambda=v_{j}\left(x, t_{r}\right)}}{\beta_{0} \delta_{0}^{2} v_{x} \prod_{\substack{k=1 \\
k \neq j}}^{3 n+1}\left(v_{j}\left(x, t_{r}\right)-v_{k}\left(x, t_{r}\right)\right)}, \\
\xi_{j, x}\left(x, t_{r}\right)=\frac{-\left.V_{32}^{(n)}\left(3 y^{2}-2 R_{m} y+S_{m}\right)\right|_{\lambda=\xi_{j}\left(x, t_{r}\right)}}{\beta_{0} \delta_{0}^{2} w \prod_{\substack{k=1 \\
k \neq j}}^{3 n+1}\left(\xi_{j}\left(x, t_{r}\right)-\xi_{k}\left(x, t_{r}\right)\right)} \\
\xi_{j, t_{r}}\left(x, t_{r}\right)=\frac{\left.\left(V_{31}^{(n)} \widetilde{V}_{32}^{(r)}-\widetilde{V}_{31}^{(r)} V_{32}^{(n)}\right)\left(3 y^{2}-2 R_{m} y+S_{m}\right)\right|_{\lambda=\xi_{j}\left(x, t_{r}\right)}}{\beta_{0} \delta_{0}^{2} w^{2} \prod_{\substack{k=1 \\
k \neq j}}^{3 n+1}\left(\xi_{j}\left(x, t_{r}\right)-\xi_{k}\left(x, t_{r}\right)\right)}
\end{gathered}
$$

with $1 \leq j \leq 3 n+1$.

Now we turn to consider expression (3.20) from which one can obtain the subsequent proposition.

Proposition 4.1. Let $P=(\lambda, y) \in \mathscr{K}_{m-1} \backslash\left\{P_{\infty_{1}}, P_{\infty_{2}}\right\},\left(x, x_{0}, t_{r}, t_{0, r}\right) \in \mathbb{C}^{4}$. Then $\psi_{2}\left(P, x, x_{0}, t_{r}, t_{0, r}\right)$ on $\mathscr{K}_{m-1} \backslash\left\{P_{\infty_{1}}, P_{\infty_{2}}\right\}$ has $3 n+1$ zeros and $3 n+1$ poles which are $\hat{\mu}_{1}\left(x, t_{r}\right), \ldots, \hat{\mu}_{3 n+1}\left(x, t_{r}\right)$ and $\hat{\mu}_{1}\left(x_{0}, t_{0, r}\right), \ldots, \hat{\mu}_{3 n+1}\left(x_{0}, t_{0, r}\right)$, respectively.

Proof. By using (4.3), (4.11) and (4.23), we can compute that

$$
\begin{aligned}
& \phi_{2}\left(P, x, t_{r}\right)=\frac{y^{2} V_{23}^{(n)}-y\left(R_{m} V_{23}^{(n)}+A_{m}\right)+B_{m}}{E_{m-1}} \\
= & \frac{1}{E_{m-1}}\left\{y^{2} V_{23}^{(n)}-y\left(R_{m} V_{23}^{(n)}+A_{m}\right)+\frac{1}{3}\left(E_{m-1, x}+u E_{m-1}+R_{m} A_{m}+2 V_{23}^{(n)} S_{m}\right)\right\} \\
= & \frac{1}{3} \frac{E_{m-1, x}}{E_{m-1}}+\frac{1}{E_{m-1}}\left\{\frac{2}{3} V_{23}^{(n)}\left(3 y^{2}-2 R_{m} y+S_{m}\right)+V_{23}^{(n)}\left(\frac{R_{m}}{3}-y\right)\left(y+\frac{A_{m}}{V_{23}^{(n)}}\right)\right\}+\frac{1}{3} u \\
= & -\frac{\mu_{j, x}\left(x, t_{r}\right)}{\lambda-\mu_{j}\left(x, t_{r}\right)}+O(1)=\partial_{x} \ln \left(\lambda-\mu_{j}\left(x, t_{r}\right)\right)+O(1), \quad \lambda \rightarrow \mu_{j}\left(x, t_{r}\right) .
\end{aligned}
$$

On the other hand, since

$$
\frac{1}{v\left(x, t_{r}\right)}\left(\phi_{2, x}\left(P, x, t_{r}\right)+\phi_{2}^{2}\left(P, x, t_{r}\right)-u\left(x, t_{r}\right) \phi_{2}\left(P, x, t_{r}\right)-\lambda\right)=\frac{\phi_{2}\left(P, x, t_{r}\right)}{\phi_{3}\left(P, x, t_{r}\right)},
$$

we can similarly derive that

$$
\begin{aligned}
& \widetilde{V}_{21}^{(r)}\left(\lambda, x, t_{r}\right) \phi_{2}\left(P, x, t_{r}\right)+\lambda \widetilde{V}_{22}^{(r)}\left(\lambda, x, t_{r}\right)+\widetilde{V}_{23}^{(r)} \frac{\phi_{2}\left(P, x, t_{r}\right)}{\phi_{3}\left(P, x, t_{r}\right)} \\
& \quad=-\frac{\mu_{j, t_{r}}\left(x, t_{r}\right)}{\lambda-\mu_{j}\left(x, t_{r}\right)}+O(1)=\partial_{t_{r}} \ln \left(\lambda-\mu_{j}\left(x, t_{r}\right)\right)+O(1), \quad \lambda \rightarrow \mu_{j}\left(x, t_{r}\right) .
\end{aligned}
$$

Substituting expressions (4.42) and (4.44) into (3.20) yields the proposition. 


\section{Quasi-periodic solutions}

In this section, we shall construct the Riemann theta function representations for the BakerAkhiezer function $\psi_{2}\left(P, x, x_{0}, t_{r}, t_{0, r}\right)$ and two meromorphic functions $\phi_{2}\left(P, x, t_{r}\right), \phi_{3}\left(P, x, t_{r}\right)$, and in particular, that of solutions for the entire long wave-short wave type hierarchy.

We denote the period lattice $\mathscr{T}_{m-1}=\left\{\underline{z} \in \mathbb{C}^{m-1} \mid \underline{z}=\underline{N}+\underline{L} \tau, \quad \underline{N}, \underline{L} \in \mathbb{Z}^{m-1}\right\}$. The complex torus $\mathscr{J}_{m-1}=\mathbb{C}^{m-1} / \mathscr{T}_{m-1}$ is called the Jacobian variety of $\mathscr{K}_{m-1}$. An Abel map $\underline{\mathscr{A}}: \mathscr{K}_{m-1} \rightarrow \mathscr{J}_{m-1}$ is defined as

$$
\underline{\mathscr{A}}(P)=\left(\int_{Q_{0}}^{P} \omega_{1}, \ldots, \int_{Q_{0}}^{P} \omega_{m-1}\right) \quad\left(\bmod \mathscr{T}_{m-1}\right)
$$

with the natural linear extension to the factor $\operatorname{group} \operatorname{Div}\left(\mathscr{K}_{m-1}\right)$

$$
\underline{\mathscr{A}}\left(\sum n_{k} P_{k}\right)=\sum n_{k} \underline{\mathscr{A}}\left(P_{k}\right)
$$

Define

$$
\begin{aligned}
& \underline{\rho}^{(1)}\left(x, t_{r}\right)=\underline{\mathscr{A}}\left(\sum_{k=1}^{3 n+1} \hat{\mu}_{k}\left(x, t_{r}\right)\right)=\sum_{k=1}^{3 n+1} \int_{Q_{0}}^{\hat{\mu}_{k}\left(x, t_{r}\right)} \underline{\omega}, \\
& \underline{\rho}^{(2)}\left(x, t_{r}\right)=\underline{\mathscr{A}}\left(\sum_{k=1}^{3 n+1} \hat{v}_{k}\left(x, t_{r}\right)\right)=\sum_{k=1}^{3 n+1} \int_{Q_{0}}^{\hat{v}_{k}\left(x, t_{r}\right)} \underline{\omega}, \\
& \underline{\rho}^{(3)}\left(x, t_{r}\right)=\underline{\mathscr{A}}\left(\sum_{k=1}^{3 n+1} \hat{\xi}_{k}\left(x, t_{r}\right)\right)=\sum_{k=1}^{3 n+1} \int_{Q_{0}}^{\hat{\xi}_{k}\left(x, t_{r}\right)} \underline{\omega},
\end{aligned}
$$

where $\underline{\rho}^{(1)}\left(x, t_{r}\right), \underline{\rho}^{(2)}\left(x, t_{r}\right), \underline{\rho}^{(3)}\left(x, t_{r}\right)$ can be linearized on $\mathscr{J}_{m-1}$ in the following text.

Let $\theta(\underline{z})$ denote the Riemann theta function associated with $\mathscr{K}_{m-1}$ equipped with homology basis and holomorphic differentials as before:

$$
\theta(\underline{z})=\sum_{\underline{N} \in \mathbb{Z}^{m-1}} \exp \{\pi i<\underline{N} \tau, \underline{N}>+2 \pi i<\underline{N}, \underline{z}>\}
$$

where $\underline{z}=\left(z_{1}, \ldots, z_{m-1}\right) \in \mathbb{C}^{m-1}$ is a complex vector, the diamond brackets denote the Euclidean scalar product:

$$
<\underline{N}, \underline{z}>=\sum_{i=1}^{m-1} N_{i} z_{i}, \quad<\underline{N} \tau, \underline{N}>=\sum_{i, j=1}^{m-1} \tau_{i j} N_{i} N_{j} .
$$

Expression (5.4) implies that

$$
\theta(\underline{z}+\underline{N}+\underline{M} \tau)=\exp \{-\pi i<\underline{M} \tau, \underline{M}>-2 \pi i<\underline{M}, \underline{z}>\} \theta(\underline{z}) .
$$

For brevity, define the function $\underline{z}: \mathscr{K}_{m-1} \times \sigma^{m-1} \mathscr{K}_{m-1} \rightarrow \mathbb{C}^{m-1}$ by

$$
\begin{aligned}
\underline{z}(P, \underline{Q}) & =\underline{M}-\underline{\mathscr{A}}(P)+\sum_{Q^{\prime} \in \underline{Q}} \mathscr{D}\left(Q^{\prime}\right) \underline{\mathscr{A}}\left(Q^{\prime}\right), \quad P \in \mathscr{K}_{m-1}, \\
\underline{Q} & =\left(Q_{1}, \ldots, Q_{m-1}\right) \in \sigma^{m-1} \mathscr{K}_{m-1},
\end{aligned}
$$


where $\sigma^{m-1} \mathscr{K}_{m-1}$ denotes the $(m-1)$-th symmetric power of $\mathscr{K}_{m-1}$ and $\underline{M}=\left(M_{1}, \ldots, M_{m-1}\right)$ is the vector of Riemann constant depending on the base point $Q_{0}$ by the following expression

$$
M_{j}=\frac{1}{2}\left(1+\tau_{j j}\right)-\sum_{\substack{l=1 \\ l \neq j}}^{m-1} \int_{\mathrm{a}_{l}} \omega_{l}(P) \int_{Q_{0}}^{P} \omega_{j}, \quad j=1, \ldots, m-1 .
$$

Then we have

$$
\begin{array}{ll}
\theta\left(\underline{z}\left(P, \hat{\mu}\left(x, t_{r}\right)\right)\right)=\theta\left(\underline{M}-\underline{\mathscr{A}}(P)+\underline{\rho}^{(1)}\left(x, t_{r}\right)\right), & P \in \mathscr{K}_{m-1}, \\
\theta\left(\underline{z}\left(P, \hat{\hat{v}}\left(x, t_{r}\right)\right)\right)=\theta\left(\underline{M}-\underline{\mathscr{A}}(P)+\underline{\rho}^{(2)}\left(x, t_{r}\right)\right), & P \in \mathscr{K}_{m-1}, \\
\theta\left(\underline{z}\left(P, \underline{\hat{\xi}}\left(x, t_{r}\right)\right)\right)=\theta\left(\underline{M}-\underline{\mathscr{A}}(P)+\underline{\rho}^{(3)}\left(x, t_{r}\right)\right), & P \in \mathscr{K}_{m-1} .
\end{array}
$$

According to divisors as seen in (4.14), (4.15) of the meromorphic functions $\phi_{2}\left(x, t_{r}\right)$ and $\phi_{3}\left(x, t_{r}\right)$, we need to introduce Abelian differentials of the third kind for their representations in terms of Riemann theta function.

Let $\omega_{Q_{1}, Q_{2}}^{(3)}(P)$ denote the normalized Abelian differential of the third kind holomorphic on $\mathscr{K}_{m-1} \backslash\left\{Q_{1}, Q_{2}\right\}$ and having simple poles at $Q_{l}$ with residues $(-1)^{l+1}, l=1,2$, then

$$
\int_{\mathrm{a}_{k}} \omega_{Q_{1}, Q_{2}}^{(3)}(P)=0, \quad \int_{\mathbb{b}_{k}} \omega_{Q_{1}, Q_{2}}^{(3)}(P)=2 \pi i \int_{Q_{2}}^{Q_{1}} \omega_{k}, k=1, \ldots, 3 n+1 .
$$

Especially, we introduce $\omega_{P_{0}, P_{\infty_{1}}}^{(3)}(P)$ and $\omega_{P_{\infty_{2}}, P_{\infty_{1}}}^{(3)}(P)$ as follows

$$
\begin{gathered}
\omega_{P_{\infty_{2}}, P_{\infty_{1}}}^{(3)}(P)=\frac{\delta_{0}\left(2 y(P)-R_{m}(\lambda)\right) \lambda^{n} d \lambda}{2\left(3 y^{2}(P)-2 R_{m}(\lambda) y(P)+S_{m}(\lambda)\right)}+\sum_{j=1}^{3 n+1} \gamma_{j} \varpi_{j}, \\
\omega_{P_{0}, P_{\infty_{1}}}^{(3)}(P)=\frac{\delta_{0}\left(2 y(P)-R_{m}(\lambda)\right) \lambda^{n} d \lambda}{2\left(3 y^{2}(P)-2 R_{m}(\lambda) y(P)+S_{m}(\lambda)\right)}+\frac{\left(y^{2}(P)-R_{m}(\lambda) y(P)+S_{m}(\lambda)\right) d \lambda}{\lambda\left(3 y^{2}(P)-2 R_{m}(\lambda) y(P)+S_{m}(\lambda)\right)}+\sum_{j=1}^{3 n+1} \eta_{j} \varpi_{j},
\end{gathered}
$$

where the $\gamma_{j}, \eta_{j}, j=1, \ldots, 3 n+1$, are uniquely determined by the requirement of normalized condition, that is vanishing a-periods

$$
\int_{\mathrm{a}_{k}} \omega_{P_{\infty_{2}}, P_{\infty_{1}}}^{(3)}(P)=0, \quad \int_{\mathrm{a}_{k}} \omega_{P_{0}, P_{\infty_{1}}}^{(3)}(P)=0
$$

From (5.11) and (5.12), we can directly calculate that

$$
\begin{gathered}
\omega_{P_{0}, P_{\infty_{1}}}^{(3)}(P) \underset{\zeta \rightarrow 0}{=}\left\{\begin{array}{l}
\left(\zeta^{-1}+O(1)\right) d \zeta, \text { as } P \rightarrow P_{0}, \quad \zeta=\lambda, \\
\left(-\zeta^{-1}+\Lambda+O(\zeta)\right) d \zeta, \text { as } P \rightarrow P_{\infty_{1}}, \\
O(1) d \zeta, \text { as } P \rightarrow P_{\infty_{2}}, \quad \zeta=\lambda^{-1},
\end{array} \quad \zeta=\lambda^{-1 / 2},\right. \\
\omega_{P_{\infty_{2}}, P_{\infty_{1}}}^{(3)}(P) \underset{\zeta \rightarrow 0}{=}\left\{\begin{array}{l}
\left(-\zeta^{-1}+O(1)\right) d \zeta, \text { as } P \rightarrow P_{\infty_{1}}, \quad \zeta=\lambda^{-1 / 2}, \\
\left(\zeta^{-1}+O(1)\right) d \zeta, \text { as } P \rightarrow P_{\infty_{2}}, \quad \zeta=\lambda^{-1},
\end{array}\right.
\end{gathered}
$$


where $\Lambda=\frac{1}{2 \beta_{0} \delta_{0}}\left(\alpha_{0} \delta_{0}+2 \beta_{0}^{2}-2 \eta_{2 n+1}-2 \delta_{0} \eta_{3 n+1}\right)$. Then we have

$$
\int_{Q_{0}}^{P} \omega_{P_{0}, P_{\infty_{1}}}^{(3)}(P) \underset{\zeta \rightarrow 0}{=}\left\{\begin{array}{l}
\ln \zeta+e_{1,0}^{(3)}\left(Q_{0}\right)+O(\zeta), \text { as } P \rightarrow P_{0}, \quad \zeta=\lambda, \\
-\ln \zeta+e_{1, \infty_{1}}^{(3)}\left(Q_{0}\right)+\Lambda \zeta+O\left(\zeta^{2}\right), \text { as } \quad P \rightarrow P_{\infty_{1}}, \quad \zeta=\lambda^{-1 / 2} \\
e_{1, \infty_{2}}^{(3)}\left(Q_{0}\right)+O(\zeta), \text { as } P \rightarrow P_{\infty_{2}}, \quad \zeta=\lambda^{-1},
\end{array}\right.
$$

and

$$
\int_{Q_{0}}^{P} \omega_{P_{\infty_{2}}, P_{\infty_{1}}}^{(3)}(P) \underset{\zeta \rightarrow 0}{=}\left\{\begin{array}{l}
-\ln \zeta+e_{2, \infty_{1}}^{(3)}\left(Q_{0}\right)+O(\zeta), \text { as } P \rightarrow P_{\infty_{1}}, \quad \zeta=\lambda^{-1 / 2} \\
\ln \zeta+e_{2, \infty_{2}}^{(3)}\left(Q_{0}\right)+O(\zeta), \text { as } P \rightarrow P_{\infty_{2}}, \quad \zeta=\lambda^{-1}
\end{array}\right.
$$

where $e_{1,0}^{(3)}\left(Q_{0}\right), e_{1, \infty_{1}}^{(3)}\left(Q_{0}\right), e_{1, \infty_{2}}^{(3)}\left(Q_{0}\right), e_{2, \infty_{1}}^{(3)}\left(Q_{0}\right), e_{2, \infty_{2}}^{(3)}\left(Q_{0}\right)$ are integration constants.

Given the asymptotic expansions at $P_{\infty_{1}}, P_{\infty_{2}}$ as in lemma 3.1, lemma 3.2 and the divisors $\left(\phi_{2}\left(P, x, t_{r}\right)\right),\left(\phi_{3}\left(P, x, t_{r}\right)\right), \mathscr{D}=\hat{\mu}_{1}\left(x_{0}, t_{0, r}\right)+\cdots+\hat{\mu}_{3 n+1}\left(x_{0}, t_{0, r}\right)$ as simple poles on $\mathscr{K}_{m-1} \backslash$ $\left\{P_{\infty_{1}}, P_{\infty_{2}}\right\}$ of $\psi_{2}\left(P, x, x_{0}, t_{r}, t_{0, r}\right)$, the representations of $\phi_{2}\left(P, x, t_{r}\right), \phi_{3}\left(P, x, t_{r}\right)$ and $\psi_{2}\left(P, x, x_{0}, t_{r}, t_{0, r}\right)$ can be uniquely determined in the following theorem, as well as those of potentials $u\left(x, t_{r}\right), v\left(x, t_{r}\right)$, $w\left(x, t_{r}\right)$.

Theorem 5.1. Let $P=(\lambda, y) \in \mathscr{K}_{m-1} \backslash\left\{P_{\infty_{1}}, P_{\infty_{2}}\right\}$ and let $\left(x_{0}, t_{0, r}\right) \in \mathbb{C}^{2},\left(x, t_{r}\right) \in \Omega_{\mu} \subseteq \mathbb{C}^{2}$, where $\Omega_{\mu}$ is open and connected. Suppose that $\mathscr{D}_{\hat{\underline{\mu}}\left(x, t_{r}\right)}$ or $\mathscr{D}_{\underline{\hat{\hat{v}}}\left(x, t_{r}\right)}$ or $\mathscr{D}_{\hat{\xi}\left(x, t_{r}\right)}$ is nonspecial for $\left(x, t_{r}\right) \in \Omega_{\mu}$. Then $\phi_{2}\left(P, x, t_{r}\right), \phi_{3}\left(P, x, t_{r}\right)$ and $\psi_{2}\left(P, x, x_{0}, t_{r}, t_{0, r}\right)$ have the following representations

$$
\begin{gathered}
\phi_{2}\left(P, x, t_{r}\right)=\frac{\theta\left(\underline{z}\left(P, \underline{\hat{v}}\left(x, t_{r}\right)\right)\right) \theta\left(\underline{z}\left(P_{\infty_{1}}, \hat{\mu}\left(x, t_{r}\right)\right)\right)}{\theta\left(\underline{z}\left(P_{\infty_{1}}, \underline{\hat{v}}\left(x, t_{r}\right)\right)\right) \theta\left(\underline{z}\left(P, \underline{\hat{\mu}}\left(x, t_{r}\right)\right)\right)} \exp \left(\int_{Q_{0}}^{P} \omega_{P_{0}, P_{\infty_{1}}}^{(3)}(P)-e_{1, \infty_{1}}^{(3)}\left(Q_{0}\right)\right), \\
\phi_{3}\left(P, x, t_{r}\right)=-v_{x}\left(x, t_{r}\right) \frac{\theta\left(\underline{z}\left(P, \underline{\hat{v}}\left(x, t_{r}\right)\right)\right) \theta\left(\underline{z}\left(P_{\infty_{2}}, \underline{\hat{\xi}}\left(x, t_{r}\right)\right)\right)}{\theta\left(\underline{z}\left(P_{\infty_{2}}, \underline{\hat{v}}\left(x, t_{r}\right)\right)\right) \theta\left(\underline{z}\left(P, \underline{\hat{\xi}}\left(x, t_{r}\right)\right)\right)} \exp \left(\int_{Q_{0}}^{P} \omega_{P_{\infty_{2}}, P_{\infty_{1}}}^{(3)}(P)-e_{2, \infty_{2}}^{(3)}\left(Q_{0}\right)\right), \\
\psi_{2}\left(P, x, x_{0}, t_{r}, t_{0, r}\right)=\exp \left(\frac{1}{2} \partial^{-1} u\left(x, t_{r}\right)-\frac{1}{2} \partial^{-1} u\left(x_{0}, t_{0, r}\right)\right) \frac{\theta\left(\underline{z}\left(P, \hat{\mu}\left(x, t_{r}\right)\right)\right) \theta\left(\underline{z}\left(P_{\infty_{1}}, \underline{\hat{\mu}}\left(x_{0}, t_{0, r}\right)\right)\right)}{\theta\left(\underline{z}\left(P_{\infty_{1}}, \hat{\mu}\left(x, t_{r}\right)\right)\right) \theta\left(\underline{z}\left(P, \hat{\mu}\left(x_{0}, t_{0, r}\right)\right)\right)} \\
\times \exp \left(\left(e_{1}^{(2)}\left(Q_{0}\right)-\int_{Q_{0}}^{P} \Omega_{2}^{(2)}(P)\right)\left(x-x_{0}\right)+\left(\tilde{e}_{1}^{(2)}\left(Q_{0}\right)-\int_{Q_{0}}^{P} \widetilde{\Omega}_{2 r+3}^{(2)}(P)\right)\left(t_{r}-t_{0, r}\right)\right),
\end{gathered}
$$

and potentials $u\left(x, t_{r}\right), v\left(x, t_{r}\right), w\left(x, t_{r}\right)$ are of the form

$$
\begin{gathered}
u\left(x, t_{r}\right)=2 \partial_{x} \ln \frac{\theta\left(\underline{z}\left(P_{\infty_{1}}, \underline{\hat{\mu}}\left(x, t_{r}\right)\right)\right)}{\theta\left(\underline{z}\left(P_{\infty_{1}}, \underline{\hat{\hat{v}}}\left(x, t_{r}\right)\right)\right)}+2 \Lambda \\
v\left(x, t_{r}\right)=v\left(x_{0}, t_{0, r}\right) \exp \left(\frac{1}{2} \partial^{-1} u\left(x, t_{r}\right)-\frac{1}{2} \partial^{-1} u\left(x_{0}, t_{0, r}\right)\right) \frac{\theta\left(\underline{z}\left(P_{\infty_{2}}, \hat{\mu}\left(x, t_{r}\right)\right)\right) \theta\left(\underline{z}\left(P_{\infty_{1}}, \hat{\mu}\left(x_{0}, t_{0, r}\right)\right)\right)}{\theta\left(\underline{z}\left(P_{\infty_{1}}, \underline{\hat{\mu}}\left(x, t_{r}\right)\right)\right) \theta\left(\underline{z}\left(P_{\infty_{2}}, \hat{\underline{\mu}}\left(x_{0}, t_{0, r}\right)\right)\right)} \\
\times \exp \left(\left(e_{1}^{(2)}\left(Q_{0}\right)-e_{2}^{(2)}\left(Q_{0}\right)\right)\left(x-x_{0}\right)+\left(\tilde{e}_{1}^{(2)}\left(Q_{0}\right)-\tilde{e}_{2}^{(2)}\left(Q_{0}\right)-\tilde{\alpha}_{r}\right)\left(t_{r}-t_{0, r}\right)\right) \\
w\left(x, t_{r}\right)=-\frac{1}{v_{x}\left(x, t_{r}\right)} \frac{\theta\left(\underline{z}\left(P_{\infty_{2}}, \underline{\hat{v}}\left(x, t_{r}\right)\right)\right) \theta\left(\underline{z}\left(P_{\infty_{1}}, \underline{\hat{\xi}}\left(x, P_{\infty_{1}}, \underline{\hat{v}}\left(x, t_{r}\right)\right)\right) \theta\left(\underline{z}\left(P_{\infty_{2}}, \underline{\hat{\xi}}\left(x, t_{r}\right)\right)\right)\right.}{\exp \left(e_{2, \infty_{2}}^{(3)}-e_{2, \infty_{1}}^{(3)}\right)}
\end{gathered}
$$


where the paths of integration in the integrals and in the Abel mapping are the same.

Proof. Assume temporarily that $\mu_{j}\left(x, t_{r}\right) \neq \mu_{j^{\prime}}\left(x, t_{r}\right)$ for $j \neq j^{\prime}$ and $\left(x, t_{r}\right) \in \widetilde{\Omega}_{\mu} \subseteq \Omega_{\mu}$, where $\widetilde{\Omega}_{\mu}$ is open and connected. Let the right hand side of (5.18) be denoted by $\Phi_{2}$. Noting (4.15) and (5.14), we can see that $\phi_{2}$ and $\Phi_{2}$ have the identical $3 n+2$ simple poles $P_{\infty_{1}}, \hat{\mu}_{1}\left(x, t_{r}\right), \cdots, \hat{\mu}_{3 n+1}\left(x, t_{r}\right)$ and simple zeros $P_{0}, \hat{v}_{1}\left(x, t_{r}\right), \cdots, \hat{v}_{3 n+1}\left(x, t_{r}\right)$. Since the arithmetic genus of the Riemann surface is $3 n+1$, utilizing the Riemann-Roch theorem, we conclude that the holomorphic function $\frac{\Phi_{2}}{\phi_{2}}=\gamma$, a constant with respect to $P$. Using (3.12) and (5.14), we can derive that

$$
\frac{\Phi_{2}}{\phi_{2}} \underset{\zeta \rightarrow 0}{=} \frac{(1+O(\zeta))\left(\zeta^{-1}+O(1)\right)}{\zeta^{-1}+O(1)} \underset{\zeta \rightarrow 0}{=} 1+O(\zeta), \quad \text { as } P \rightarrow P_{\infty_{1}}, \quad \zeta=\lambda^{-1 / 2},
$$

which yields that $\gamma=1$. Similarly, we can prove expression (5.19). The asymptotic expansions of the Baker-Akhiezer function $\psi_{2}\left(P, x, x_{0}, t_{r}, t_{0, r}\right)$ in lemma 3.2 and divisor in proposition 4.1 reveal that $\psi_{2}\left(P, x, x_{0}, t_{r}, t_{0, r}\right)$ has the Riemann theta representation (5.20), where the function $u\left(x, t_{r}\right)$ can be determined later. With help of a meromorphic differential

$$
\Omega\left(x, x_{0}, t_{r}, t_{0, r}\right)=\frac{\partial}{\partial \lambda} \ln \left(\psi_{2}\left(P, x, x_{0}, t_{r}, t_{0, r}\right)\right) d \lambda,
$$

the Abel map can be linearized in the following form [25]

$$
\underline{\rho}^{(j)}\left(x, t_{r}\right)=\underline{\rho}^{(j)}\left(x_{0}, t_{0, r}\right)+\underline{U}_{2}^{(2)}\left(x-x_{0}\right)+\underline{U}_{2 r+3}^{(2)}\left(t_{r}-t_{0, r}\right) \quad\left(\bmod \mathscr{T}_{m-1}\right), \quad j=1,2,3 .
$$

Therefore, $\theta\left(\underline{z}\left(P_{\infty_{1}}, \underline{\hat{\mu}}\left(x, t_{r}\right)\right)\right)$ and $\theta\left(\underline{z}\left(P_{\infty_{1}}, \underline{\hat{v}}\left(x, t_{r}\right)\right)\right)$ could be written briefly in the following form

$$
\begin{aligned}
& \theta\left(\underline{z}\left(P_{\infty_{1}}, \underline{\hat{\mu}}\left(x, t_{r}\right)\right)\right)=\theta\left(\underline{M}^{(1)}+\underline{U}_{2}^{(2)} x+\underline{\widetilde{U}}_{2 r+3}^{(2)} t_{r}\right), \\
& \theta\left(\underline{z}\left(P_{\infty_{1}}, \underline{\hat{v}}\left(x, t_{r}\right)\right)\right)=\theta\left(\underline{M}^{(2)}+\underline{U}_{2}^{(2)} x+\underline{\widetilde{U}}_{2 r+3}^{(2)} t_{r}\right)
\end{aligned}
$$

where

$$
\underline{M}^{(j)}=\underline{M}-\mathscr{A}\left(P_{\infty_{1}}\right)+\underline{\rho}^{(j)}\left(x_{0}, t_{0, r}\right)-\underline{U}_{2}^{(2)} x_{0}-\underline{\widetilde{U}}_{2 r+3}^{(2)} t_{0, r}, \quad j=1,2 .
$$

In order to derive (5.21), we expand $\phi_{2}$ of (5.18) near $P_{\infty_{1}}$ under the local coordinate $\zeta=\lambda^{-1 / 2}$

$$
\phi_{2} \underset{\zeta \rightarrow 0}{=} \zeta^{-1}+\partial_{x} \ln \frac{\theta\left(\underline{z}\left(P_{\infty_{1}}, \underline{\hat{\mu}}\left(x, t_{r}\right)\right)\right)}{\theta\left(\underline{z}\left(P_{\infty_{1}}, \underline{\hat{\hat{v}}}\left(x, t_{r}\right)\right)\right)}+\Lambda+O(\zeta)
$$

from which we can derive expression (5.21) by comparing asymptotic expansion (5.28) of $\phi_{2}$ with (3.12) in lemma 3.1. Representations for $v$ and $w$ as seen in (5.22) and (5.23) can be deduced similarly by expanding $\psi_{2}$ and $\phi_{3}$ respectively near $P_{\infty_{2}}$ and $P_{\infty_{1}}$. Observing the continuity of the Abel map $\mathscr{A}$, one can extend the result from $\left(x, t_{r}\right) \in \widetilde{\Omega}_{\mu}$ to $\left(x, t_{r}\right) \in \Omega_{\mu}$, which completes the proof of the theorem.

\section{Acknowledgments}

This work is supported by National Natural Science Foundation of China (Grant Nos. 11871440, 11501520, 11522112) and Outstanding Young Talent Research Fund of Zhengzhou University (Grant No. 1521315001). 


\section{References}

[1] M.J. Ablowitz, P.A. Clarkson, Solitons, Nonlinear Evolution Equation and Inverse Scattering (Cambridge University Press, Cambridge, 1991).

[2] H. Airault, H.P. McKean, J. Moser, Rational and elliptic solutions of the Korteweg-de Vries equation a related many-body problem, Comm. Pure Appl. Math., 30 (1977) 95-148.

[3] S.J. Alber, On finite-zone solutions of relativistic Toda lattices, Lett. Math. Phys., 17 (1989) 149-155.

[4] E.D. Belokolos, A.I. Bobenko, V.Z. Enol'skii, A.R. Its, V.B. Mateveev, Algebro-Geometric Approach to Nonlinear Integrable Equations (Springer-Verlag, Berlin, 1994).

[5] V.M. Buchstaber, V.Z. Enolskii, D.V. Leykin, Uniformization of Jacobi varieties of trigonal curves and nonlinear differential equations, Funct. Anal. Appl., 34 (2000) 159-171.

[6] R. Camassa, D.D. Holm, An integrable shallow water equation with peaked solitons, Phys. Rev. Lett., 71 (1993) 1661-1664.

[7] C.W. Cao, Y.T. Wu, X.G. Geng, Relation between the Kadomtsev-Petviashvili equation and the confocal involutive system, J. Math. Phys., 40 (1999) 3948-3970.

[8] Y. Cheng, Constraints of the Kadomtsev-Petviashvili hierarchy, J. Math. Phys., 33 (1992) 3774-3782.

[9] E. Date, S. Tanaka, Periodic multi-soliton solutions of Korteweg-de Vries equation and Toda lattice, Progr. Theoret. Phys. Suppl., 59 (1976) 107-125.

[10] A. Degasperis, M. Procesi, Asymptotic integrability, Symmetry and Perturbation Theory (World Scientific, Singapore, 1999) 23-27.

[11] A. Degtyarev, I. Itenberg, V. Zvonilov, Real trigonal curves and real elliptic surfaces of type I, J. Reine Angew. Math., 686 (2014) 221-246.

[12] R. Dickson, F. Gesztesy, K. Unterkofler, A new approach to the Boussinesq hierarchy, Math. Nachr., 198 (1999) 51-108.

[13] R. Dickson, F. Gesztesy, K. Unterkofler, Algebro-geometric solutions of the Boussinesq hierarchy, Rev. Math. Phys., 11 (1999) 823-879.

[14] B.A. Dubrovin, Theta functions and nonlinear equations, Russian Math. Surveys, 36 (1981) 11-92.

[15] M. England, Higher genus abelian functions associated with cyclic trigonal curves, SIGMA, 6 (2010) 025 .

[16] J. Fay, Theta Functions on Riemann Surfaces (Springer-Verlag, Berlin, 1973).

[17] C.S. Gardner, J.M. Greene, M.D. Kruskal, R.M. Miura, Method for solving the Korteweg-de Vries equation, Phys. Rev. Lett., 19 (1967) 1095-1097.

[18] X.G. Geng, H.H. Dai, J.Y. Zhu, Decomposition of the discrete Ablowitz-Ladik hierarchy, Stud. Appl. Math., 118 (2007) 281-312.

[19] X.G. Geng, H. Liu, The nonlinear steepest descent method to long-time asymptotics of the coupled nonlinear Schrödinger equation, J. Nonlinear Sci. 28 (2018) 739-763.

[20] X.G. Geng, L.H. Wu, G.L. He, Algebro-geometric constructions of the modified Boussinesq flows and quasi-periodic solutions, Phys. D, 240 (2011) 1262-1288.

[21] X.G. Geng, L.H. Wu, G.L. He, Quasi-periodic solutions of the Kaup-Kupershmidt hierarchy, J. Nonlinear Sci., 23 (2013) 527-555.

[22] X.G. Geng, B. Xue, A three-component generalization of Camassa-Holm equation with N-peakon solutions, Adv. Math., 226 (2011) 827-839.

[23] X.G. Geng, B. Xue, An extension of integrable peakon equations with cubic nonlinearity, Nonlinearity, 22 (2009) 1847-1856.

[24] X.G. Geng, B. Xue, Quasi-periodic solutions of mixed AKNS equations, Nonlinear Anal., 73 (2010) $3662-3674$.

[25] X.G. Geng, Y.Y. Zhai, H.H. Dai, Algebro-geometric solutions of the coupled modified Korteweg-de Vries hierarchy, Adv. Math., 263 (2014) 123-153.

[26] F. Gesztesy, H. Holden, Real-valued algebro-geometric solutions of the Camassa- Holm hierarchy, Philos. Trans. Roy. Soc. Lond. Ser. A (Math. Phys. Eng. Sci.), 366 (2008) 1025-1054.

[27] P. Griffiths, J. Harris, Principles of Algebraic Geometry (Wiley, New York, 1994).

[28] G.L. He, X.G. Geng, L.H. Wu, Algebro-geometric quasi-periodic solutions to the three-wave resonant interaction hierarchy, SIAM J. Math. Anal., 46 (2014) 1348-1384. 
[29] R. Hirota, The Direct Method in Soliton Theory (Cambridge University Press, Cambridge, 2004).

[30] A.N.W. Hone, J.P. Wang, Integrable peakon equations with cubic nonlinearity, J. Phys. A: Math. Theor., 41 (2008) 372002.

[31] D.J. Korteweg, G.de Vries, On the change of form of long waves advancing in a rectangular canal, and on a new type of long stationary wave, Philos. Mag. Ser., 39 (1895) 422-443.

[32] I.M. Krichever, Algebraic-geometric construction of the Zaharov-Sabat equations and their periodic solutions, Soviet Math. Dokl., 17 (1976) 394-397.

[33] I.M. Krichever, Integration of nonlinear equations by the methods of algebraic geometry, Funct. Anal. Appl., 11 (1977) 12-26.

[34] L.C. Li, I. Nenciu, The periodic defocusing Ablowitz-Ladik equation and the geometry of Floquet CMV matrices, Adv. Math., 231 (2012) 3330-3388.

[35] X.C. Liu, Y. Liu, Peter J. Olver, C.Z. Qu, Orbital stability of peakons for a generalization of the modified Camassa-Holm equation, Nonlinearity, 27 (2014) 2297-2319.

[36] H. Lundmark, J. Szmigielski, An inverse spectral problem related to the Geng-Xue two-component peakon equation, Mem. Amer. Math. Soc., 244 (2016), no. 1155, vii+87 pp.

[37] H. Lundmark, J. Szmigielski, Dynamics of interlacing peakons (and shockpeakons) in the Geng-Xue equation, Journal of Integrable Systems, J. Integrable Syst., 2 (2017) xyw014, 65 pp.

[38] Y.C. Ma, The complete solution of the long-wave-short-wave resonance equations, Stud. Appl. Math., 59 (1978) 201-221.

[39] W.X. Ma, Trigonal curves and algebro-geometric solutions to soliton hierarchies I, Proc. R. Soc. A, 473 (2017) 20170232

[40] W.X. Ma, Trigonal curves and algebro-geometric solutions to soliton hierarchies II, Proc. R. Soc. A, 473 (2017) 20170233.

[41] Y.C. Ma, M.J. Ablowitz, The periodic cubic Schrödinger equation, Stud. Appl. Math., 65 (1981) 113158.

[42] W.X. Ma, Y. Zhou, Lump solutions to nonlinear partial differential equations via Hirota bilinear forms, J. Differential Equations, 264 (2018) 2633-2659.

[43] H.P. McKean, Integrable systems and algebraic curves, Global Analysis, Lecture Notes in Math., 755 (Springer, Berlin, 1979) 83-200.

[44] V.B. Matveev, M.A. Salle, Darboux Transformations and Solitons (Sprilinger, Berlin, 1991).

[45] V.B. Matveev, A.O. Smirnov, On the Riemann theta function of a trigonal curve and solutions of the Boussinesq and KP equations, Lett. Math. Phys., 14 (1987) 25-31.

[46] V.B. Matveev, A.O. Smirnov, Symmetric reductions of the Riemann-function and some of their applications to the Schrödinger and Boussinesq equations, Amer. Math. Soc. Transl., 157 (1993) 227-237.

[47] R.M. Miura, Korteweg-de Vries equation and generalizations I. A remarkable explicit nonlinear transformation, J. Math. Phys., 9 (1968) 1202-1204.

[48] D. Mumford, Tata lectures on theta II (Birkhäuser, Boston, 1984).

[49] Y. Ônishi, Determinant formulae in Abelian functions for a general trigonal curve of degree five, Comput. Methods Funct. Theory, 11 (2011) 547-574.

[50] R. Pego, Origin of the KdV equation, Notices Amer. Math. Soc., 45 (1998) 358.

[51] E. Previato, Hyperelliptic quasi-periodic and soliton solutions of the nonlinear Schrödinger equation, Duke Math. J., 52 (1985) 329-377.

[52] E. Previato, Monodromy of Boussinesq elliptic operators, Acta Appl. Math., 36 (1994) 49-55.

[53] E. Previato, The Calogero-Moser-Krichever system and elliptic Boussinesq solitons, Hamiltonian Systems, Transformation Groups and Spectral Transform Methods, (CRM, Monreal, 1990) 57-67.

[54] E. Previato, J.L. Verdier, Boussinesq elliptic solitons: the cyclic case, Proc. Indo-French Conf. on Geometry, Dehli, 1993 (Hindustan Book Agency, Delhi, 1993) 173-185.

[55] A.O. Smirnov, A matrix analogue of Appell's theorem and reductions of multidimensional Riemann theta-functions, Math. USSR Sbornik, 61 (1988) 379-388.

[56] N. Yajima, M. Oikawa, Formation and interaction of sonic-Langmuir solitons-inverse scattering method, Progr. Theoret. Phys., 56 (1976) 1719-1739. 